\title{
Equivalence of Littlewood-Paley square function and area function characterizations of weighted product Hardy spaces associated to operators
}

\author{
By Xuan Thinh Duong, Guorong Hu and Ji LI
}

(Received June 16, 2017)

\begin{abstract}
Let $L_{1}$ and $L_{2}$ be nonnegative self-adjoint operators acting on $L^{2}\left(X_{1}\right)$ and $L^{2}\left(X_{2}\right)$, respectively, where $X_{1}$ and $X_{2}$ are spaces of homogeneous type. Assume that $L_{1}$ and $L_{2}$ have Gaussian heat kernel bounds. This paper aims to study some equivalent characterizations of the weighted product Hardy spaces $H_{w, L_{1}, L_{2}}^{p}\left(X_{1} \times X_{2}\right)$ associated to $L_{1}$ and $L_{2}$, for $p \in(0, \infty)$ and the weight $w$ belongs to the product Muckenhoupt class $A_{\infty}\left(X_{1} \times X_{2}\right)$. Our main result is that the spaces $H_{w, L_{1}, L_{2}}^{p}\left(X_{1} \times X_{2}\right)$ introduced via area functions can be equivalently characterized by the Littlewood-Paley $g$-functions and $g_{\lambda_{1}, \lambda_{2}}^{*}$-functions, as well as the Peetre type maximal functions, without any further assumption beyond the Gaussian upper bounds on the heat kernels of $L_{1}$ and $L_{2}$. Our results are new even in the unweighted product setting.
\end{abstract}

\section{Introduction.}

The theory of Hardy spaces has been a successful story in modern harmonic analysis in the last fifty years. In the classical case of the Euclidean space $\mathbb{R}^{n}$, it is well known that among other equivalent characterizations the Hardy spaces $H^{p}\left(\mathbb{R}^{n}\right)$ can be characterized by area functions, by Littlewood-Paley $g$-functions and by atomic decomposition [12], [21]. Concerning Hardy spaces $H^{p}(X)$ on a space of homogeneous type $X$, a new approach to show the equivalence between characterizations of $H^{p}(X)$ by area functions and $g$-functions is to use the Plancherel-Polya type inequality, which requires the Hölder continuity and cancellation conditions [6]. About the more recent Hardy spaces $H_{L}^{p}(X)$ associated to an operator $L$ on a space of homogeneous type $X$, one used to need extra assumptions to show that the characterizations by area functions and by LittlewoodPaley $g$-functions are equivalent, for example, Hölder continuity was assumed in [8] and Moser type estimate in [10]. Only recently, the equivalence of the characterizations of $H_{L}^{p}(X)$ by area functions and by Littlewood-Paley $g$-functions was obtained in [16] under no further assumption beyond the Gaussian heat kernel bounds. Actually, the work in [16] was done in the weighted setting.

The aim of the current paper is to prove the equivalence between the characterizations of the weighted product Hardy spaces $H_{w, L_{1}, L_{2}}^{p}\left(X_{1} \times X_{2}\right)$ in terms of the area functions and Littlewood-Paley square functions, see Theorems 1.4 and 1.5, where we

2010 Mathematics Subject Classification. Primary 42B25; Secondary 42B30, 47B25.

Key Words and Phrases. product Hardy spaces, non-negative self-adjoint operator, heat semigroup, Littlewood-Paley functions, space of homogeneous type.

The first and third authors were supported by DP 160100153. The second author is the corresponding author. 
assume only that the operators $L_{1}$ and $L_{2}$ are nonnegative self-adjoint and have Gaussian upper bounds on their heat kernels. This extends the main result in [16] to the product setting. The strength of our results is that not only they are new for the setting of product spaces and cover larger classes of operators $L_{1}$ and $L_{2}$ but also recover a number of known results whose proofs rely on extra regularity of the semigroups. In particular, our Theorems 1.4 and 1.5

(i) give a direct proof for the equivalent characterizations via Littlewood-Paley square functions of the classical product Hardy space by Chang-Fefferman in [5],

(ii) provide a new proof of equivalent characterizations via Littlewood-Paley square functions of the product Hardy spaces on spaces of homogeneous type in [15] whose proofs require the Hölder continuity and cancellation conditions,

(iii) provide the missing characterizations of product Hardy spaces via LittlewoodPaley square functions in the setting developed in [7] and [10], and

(iv) recover the recent related known results in the setting of Bessel operators in [9] whose proofs relied on the Hölder regularity, and results for Bessel Schrödinger operators in [1] whose proofs used the Moser type inequality.

For more details and explanations of (iii) and (iv), we refer to Section 4.

We now recall some basic facts concerning spaces of homogeneous type. Let $(X, \rho)$ be a metric space, and $\mu$ be a positive Radon measure on $X$. Write $V(x, r):=\mu(B(x, r))$, where $B(x, r)$ denotes the open ball centered at $x$ with radius $r$. We say that $(X, \rho, \mu)$ is a space of homogeneous type if it satisfies the volume doubling property:

$$
V(x, 2 r) \leq V(x, r)
$$

for all $x \in X$ and $r>0$. An immediate consequence of (1.1) is that there exist constants $C$ and $n$ such that

$$
V(x, \lambda r) \leq C \lambda^{n} V(x, r)
$$

for all $x \in X, r>0$ and $\lambda \geq 1$. The constant $n$ plays the role of an upper bound of the dimension, though it need not even be an integer, and we want to take $n$ as small as possible. There also exist constants $C$ and $D, 0 \leq D \leq n$, so that

$$
V(y, r) \leq C\left(1+\frac{\rho(x, y)}{r}\right)^{D} V(x, r)
$$

uniformly for all $x, y \in X$ and $r>0$. Indeed, property (1.3) with $D=n$ is a direct consequence of (1.2). In the case where $X$ is the Euclidean space $\mathbb{R}^{n}$ or a Lie group of polynomial growth, $D$ can be chosen to be 0 .

Throughout this paper, we assume that, for $i=1,2,\left(X_{i}, \rho_{i}, \mu_{i}\right)$ is a space of homogenous type with $\mu\left(X_{i}\right)=\infty$. The constant $n$ (resp. D) in (1.2) (resp. (1.3)) for $\left(X_{i}, \rho_{i}, \mu_{i}\right)$ is denoted by $n_{i}$ (resp. $\left.D_{i}\right)$. Let $L_{i}, i=1,2$, be a linear operator on $L^{2}\left(X_{i}, d \mu_{i}\right)$ satisfying the following properties:

(H1) Each $L_{i}$ is a nonnegative self-adjoint operator on $L^{2}\left(X_{i}, d \mu_{i}\right)$;

(H2) The kernel of the semigroup $e^{-t L_{i}}$, denoted by $p_{t}^{(i)}\left(x_{i}, y_{i}\right)$, is a measurable function on $X_{i} \times X_{i}$ and obeys a Gaussian upper bound, that is, 


$$
\left|p_{t}^{(i)}\left(x_{i}, y_{i}\right)\right| \leq \frac{C_{i}}{V\left(x_{i}, \sqrt{t}\right)} \exp \left(-\frac{\rho_{i}\left(x_{i}, y_{i}\right)^{2}}{c_{i} t}\right)
$$

for all $t>0$ and a.e. $\left(x_{i}, y_{i}\right) \in X_{i} \times X_{i}$, where $C_{i}$ and $c_{i}$ are positive constants, for $i=1,2$.

DeFinition 1.1. Let $\Phi_{1}, \Phi_{2} \in \mathcal{S}(\mathbb{R})$.

a) Given a function $f \in L^{2}\left(X_{1} \times X_{2}\right)$, we define the product type Littlewood-Paley $g$-function $g_{\Phi_{1}, \Phi_{2}, L_{1}, L_{2}}(f)$ associated to $L_{1}$ and $L_{2}$ by

$$
g_{\Phi_{1}, \Phi_{2}, L_{1}, L_{2}}(f)\left(x_{1}, x_{2}\right):=\left(\int_{0}^{\infty} \int_{0}^{\infty}\left|\Phi_{1}\left(t_{1} \sqrt{L_{1}}\right) \otimes \Phi_{2}\left(t_{2} \sqrt{L_{2}}\right) f\left(x_{1}, x_{2}\right)\right|^{2} \frac{d t_{1}}{t_{1}} \frac{d t_{2}}{t_{2}}\right)^{1 / 2} .
$$

b) The product type area function $S_{\Phi_{1}, \Phi_{2}, L_{1}, L_{2}}(f)$ associated to $L_{1}$ and $L_{2}$ is defined by

$$
\begin{aligned}
& S_{\Phi_{1}, \Phi_{2}, L_{1}, L_{2}}(f)\left(x_{1}, x_{2}\right) \\
& \quad:=\left(\iint_{\Gamma_{1}\left(x_{1}\right) \times \Gamma_{2}\left(x_{2}\right)}\left|\Phi_{1}\left(t_{1} \sqrt{L_{1}}\right) \otimes \Phi_{2}\left(t_{2} \sqrt{L_{2}}\right) f\left(y_{1}, y_{2}\right)\right|^{2} \frac{d \mu_{1}\left(y_{1}\right) d t_{1}}{V\left(x_{1}, t_{1}\right) t_{1}} \frac{d \mu_{2}\left(y_{2}\right) d t_{2}}{V\left(x_{2}, t_{2}\right) t_{2}}\right)^{1 / 2},
\end{aligned}
$$

where $\Gamma_{i}\left(x_{i}\right):=\left\{\left(y_{i}, t_{i}\right) \in X_{i} \times(0, \infty): \rho_{i}\left(x_{i}, y_{i}\right)<t_{i}\right\}$ for $i=1,2$.

c) For $\lambda_{1}, \lambda_{2}, t_{1}, t_{2}>0$, the product Peetre type maximal function associated to $L_{1}$ and $L_{2}$ is defined by

$$
\begin{aligned}
& {\left[\Phi_{1}\left(t_{1} \sqrt{L_{1}}\right) \otimes \Phi_{2}\left(t_{2} \sqrt{L_{2}}\right)\right]_{\lambda_{1}, \lambda_{2}}^{*} f\left(x_{1}, x_{2}\right)} \\
& \quad:=\sup _{\left(y_{1}, y_{2}\right) \in X_{1} \times X_{2}} \frac{\left|\Phi_{1}\left(t_{1} \sqrt{L_{1}}\right) \otimes \Phi_{2}\left(t_{2} \sqrt{L_{2}}\right) f\left(y_{1}, y_{2}\right)\right|}{\left(1+t_{1}^{-1} \rho_{1}\left(x_{1}, y_{1}\right)\right)^{\lambda_{1}}\left(1+t_{2}^{-1} \rho_{2}\left(x_{2}, y_{2}\right)\right)^{\lambda_{2}}},
\end{aligned}
$$

for $\left(x_{1}, x_{2}\right) \in X_{1} \times X_{2}$.

d) For $\lambda_{1}, \lambda_{2}>0$, the product type Littlewood-Paley $g_{\lambda_{1}, \lambda_{2}}^{*}$-function associated to $L_{1}$ and $L_{2}$ is defined by

$g_{\Phi_{1}, \Phi_{2}, L_{1}, L_{2}, \lambda_{1}, \lambda_{2}}^{*}(f)\left(x_{1}, x_{2}\right)$

$:=\left(\int_{0}^{\infty} \int_{0}^{\infty} \int_{X_{1}} \int_{X_{2}} \frac{\left|\Phi_{1}\left(t_{1} \sqrt{L_{1}}\right) \otimes \Phi_{2}\left(t_{2} \sqrt{L_{2}}\right) f\left(y_{1}, y_{2}\right)\right|^{2}}{\left(1+t_{1}^{-1} \rho_{1}\left(x_{1}, y_{1}\right)\right)^{n_{1} \lambda_{1}}\left(1+t_{2}^{-1} \rho_{2}\left(x_{2}, y_{2}\right)\right)^{n_{2} \lambda_{2}}} \frac{d \mu_{1}\left(y_{1}\right) d t_{1}}{V\left(x_{1}, t_{1}\right) t_{1}} \frac{d \mu_{2}\left(y_{2}\right) d t_{2}}{V\left(x_{2}, t_{2}\right) t_{2}}\right)^{1 / 2}$,

for $\left(x_{1}, x_{2}\right) \in X_{1} \times X_{2}$.

Following [13], [14], we introduce product Muckenhoupt weights on spaces of homogeneous type.

Definition 1.2. A nonnegative locally integrable function $w$ on $X_{1} \times X_{2}$ is said to belong to the product Muckenhoupt class $A_{p}\left(X_{1} \times X_{2}\right)$ for a given $p \in(1, \infty)$, if there is a constant $C$ such that for all balls $B_{1} \subset X_{1}$ and $B_{2} \subset X_{2}$, 


$$
\begin{aligned}
& \left(\frac{1}{\mu_{1}\left(B_{1}\right) \mu_{2}\left(B_{2}\right)} \iint_{B_{1} \times B_{2}} w\left(x_{1}, x_{2}\right) d \mu_{1}\left(x_{1}\right) d \mu_{2}\left(x_{2}\right)\right) \\
& \quad \times\left(\frac{1}{\mu_{1}\left(B_{1}\right) \mu_{2}\left(B_{2}\right)} \iint_{B_{1} \times B_{2}} w\left(x_{1}, x_{2}\right)^{-1 /(p-1)} d \mu_{1}\left(x_{1}\right) d \mu_{2}\left(x_{2}\right)\right)^{p-1} \leq C .
\end{aligned}
$$

The class $A_{1}\left(X_{1} \times X_{2}\right)$ is defined to be the collection of all nonnegative locally integrable functions $w$ on $X_{1} \times X_{2}$ such that

$$
\left(\frac{1}{\mu_{1}\left(B_{1}\right) \mu_{2}\left(B_{2}\right)} \iint_{B_{1} \times B_{2}} w\left(x_{1}, x_{2}\right) d \mu_{1}\left(x_{1}\right) d \mu_{2}\left(x_{2}\right)\right)\left\|w^{-1}\right\|_{L^{\infty}\left(B_{1} \times B_{2}\right)} \leq C
$$

for all balls $B_{1} \subset X_{1}$ and $B_{2} \subset X_{2}$. define

We let $A_{\infty}\left(X_{1} \times X_{2}\right):=\bigcup_{1 \leq p<\infty} A_{p}\left(X_{1} \times X_{2}\right)$ and, for any $w \in A_{\infty}\left(X_{1} \times X_{2}\right)$,

$$
q_{w}:=\inf \left\{q \in[1, \infty): w \in A_{q}\left(X_{1} \times X_{2}\right)\right\},
$$

the critical index for $w$ (see, for instance, [14]). For $1<p<\infty$, the weighted Lebesgue space $L_{w}^{p}\left(X_{1} \times X_{2}\right)$ is defined to be the collection of all measurable functions $f$ on $X_{1} \times X_{2}$ for which

$$
\|f\|_{L_{w}^{p}\left(X_{1} \times X_{2}\right)}:=\left(\iint_{X_{1} \times X_{2}}\left|f\left(x_{1}, x_{2}\right)\right|^{p} w\left(x_{1}, x_{2}\right) d \mu_{1}\left(x_{1}\right) d \mu_{2}\left(x_{2}\right)\right)^{1 / p}<\infty .
$$

We next introduce a class of functions on $\mathbb{R}$ which will play a significant role in our formulation.

Definition 1.3. A function $\Phi \in \mathcal{S}(\mathbb{R})$ is said to belong to the class $\mathcal{A}(\mathbb{R})$ if it satisfies the Tauberian condition, namely,

$$
|\Phi(\lambda)|>0 \quad \text { on }\{\varepsilon / 2<|\lambda|<2 \varepsilon\}
$$

for some $\varepsilon>0$.

Now we are ready to state our main results.

ThEOREM 1.4. Let $\Phi_{1}, \Phi_{2}, \widetilde{\Phi}_{1}, \widetilde{\Phi}_{2} \in \mathcal{A}(\mathbb{R})$ be even functions satisfying

$$
\Phi_{1}(0)=\Phi_{2}(0)=\widetilde{\Phi}_{1}(0)=\widetilde{\Phi}_{2}(0)=0 .
$$

Let $p \in(0, \infty)$ and $w \in A_{\infty}\left(X_{1} \times X_{2}\right)$. Then there exists a constant $C=$ $C\left(p, w, \Phi_{1}, \Phi_{2}, \widetilde{\Phi}_{1}, \widetilde{\Phi}_{2}\right)$ such that for all $f \in L^{2}\left(X_{1} \times X_{2}\right)$,

$$
\begin{aligned}
C^{-1}\left\|g_{\widetilde{\Phi}_{1}, \widetilde{\Phi}_{2}, L_{1}, L_{2}}(f)\right\|_{L_{w}^{p}\left(X_{1} \times X_{2}\right)} & \leq\left\|g_{\Phi_{1}, \Phi_{2}, L_{1}, L_{2}}(f)\right\|_{L_{w}^{p}\left(X_{1} \times X_{2}\right)} \\
& \leq C\left\|g_{\widetilde{\Phi}_{1}, \widetilde{\Phi}_{2}, L_{1}, L_{2}}(f)\right\|_{L_{w}^{p}\left(X_{1} \times X_{2}\right)} .
\end{aligned}
$$

TheOrem 1.5. Let $\Phi_{1}, \Phi_{2} \in \mathcal{A}(\mathbb{R})$ be even functions. Let $p \in(0, \infty), \quad \lambda_{i}>$ $2 q_{w} / \min \{p, 2\}$ and $\lambda_{i}^{\prime}>\left(n_{i}+D_{i}\right) q_{w} / \min \{p, 2\}, i=1,2$. Then for $f \in L^{2}\left(X_{1} \times X_{2}\right)$ 
we have the following quasi-norm equivalence:

$$
\begin{aligned}
& \left\|S_{\Phi_{1}, \Phi_{2}, L_{1}, L_{2}}(f)\right\|_{L_{w}^{p}\left(X_{1} \times X_{2}\right)} \sim\left\|g_{\Phi_{1}, \Phi_{2}, L_{1}, L_{2}}(f)\right\|_{L_{w}^{p}\left(X_{1} \times X_{2}\right)} \\
& \quad \sim\left\|g_{\Phi_{1}, \Phi_{2}, L_{1}, L_{2}, \lambda_{1}, \lambda_{2}}^{*}(f)\right\|_{L_{w}^{p}\left(X_{1} \times X_{2}\right)} \\
& \quad \sim\left\|\left(\int_{0}^{\infty} \int_{0}^{\infty}\left|\left[\Phi_{1}\left(t_{1} \sqrt{L_{1}}\right) \otimes \Phi_{2}\left(t_{2} \sqrt{L_{2}}\right)\right]_{\lambda_{1}^{\prime}, \lambda_{2}^{\prime}}^{*} f\right|^{2} \frac{d t_{1}}{t_{1}} \frac{d t_{2}}{t_{2}}\right)^{1 / 2}\right\|_{L_{w}^{p}\left(X_{1} \times X_{2}\right)} .
\end{aligned}
$$

Having these results, one can introduce weighted product Hardy spaces associated to $L_{1}$ and $L_{2}$ as follows:

Definition 1.6. Let $p \in(0, \infty), w \in A_{\infty}\left(X_{1} \times X_{2}\right)$, and $\Phi_{1}, \Phi_{2} \in \mathcal{A}(\mathbb{R})$ be even functions satisfying

$$
\Phi_{1}(0)=\Phi_{2}(0)=0
$$

The weighted product Hardy space $H_{w, L_{1}, L_{2}}^{p}\left(X_{1} \times X_{2}\right)$ associated to $L_{1}$ and $L_{2}$ is defined to be the completion of the set

$$
\left\{f \in L^{2}\left(X_{1} \times X_{2}\right): S_{\Phi_{1}, \Phi_{2}, L_{1}, L_{2}}(f) \in L_{w}^{p}\left(X_{1} \times X_{2}\right)\right\}
$$

with respect to the (quasi-)norm

$$
\|f\|_{H_{w, L_{1}, L_{2}}^{p}\left(X_{1} \times X_{2}\right)}:=\left\|S_{\Phi_{1}, \Phi_{2}, L_{1}, L_{2}}(f)\right\|_{L_{w}^{p}\left(X_{1} \times X_{2}\right)} .
$$

REMARK 1.7. Combining Theorems 1.4 and 1.5 we see that the definition of $H_{w, L_{1}, L_{2}}^{p}\left(X_{1} \times X_{2}\right)$ is independent of the choice of the even functions $\Phi_{1}, \Phi_{2}$, as long as $\Phi_{1}, \Phi_{2} \in \mathcal{A}(\mathbb{R})$ and satisfy $\Phi_{1}(0)=\Phi_{2}(0)=0$. In particular, if one chooses $\Phi_{1}(\lambda)=\Phi_{2}(\lambda)=\lambda^{2} e^{-\lambda^{2}}$, then the (quasi-)norm of $H_{w, L_{1}, L_{2}}^{p}\left(X_{1} \times X_{2}\right)$ can be written as

$$
\begin{aligned}
\|f\|_{H_{w, L_{1}, L_{2}}^{p}\left(X_{1} \times X_{2}\right)}:=\|\left(\iint_{\Gamma_{1}\left(x_{1}\right) \times \Gamma_{2}\left(x_{2}\right)}\left|\left(t_{1}^{2} L_{1} e^{-t_{1}^{2} L_{1}}\right) \otimes\left(t_{2}^{2} L_{2} e^{-t_{2}^{2} L_{2}}\right) f\left(y_{1}, y_{2}\right)\right|^{2}\right. \\
\left.\times \frac{d \mu_{1}\left(y_{1}\right) d t_{1}}{V\left(x_{1}, t_{1}\right) t_{1}} \frac{d \mu_{2}\left(y_{2}\right) d t_{2}}{V\left(x_{2}, t_{2}\right) t_{2}}\right)^{1 / 2} \|_{L_{w}^{p}\left(X_{1} \times X_{2}\right)} .
\end{aligned}
$$

Furthermore, from Theorem 1.5 we see that each quantity in (1.5) can be used as an equivalent (quasi-)norm of the space $H_{w, L_{1}, L_{2}}^{p}\left(X_{1} \times X_{2}\right)$.

As mentioned above, we make no further assumption on the heat kernel of $L_{1}$ or $L_{2}$ beyond the Gaussian upper bounds. Thus, the approach in [8] which uses a PlancherelPolya type inequality and the approach in $[\mathbf{1 0}]$ which uses a discrete characterization can not be applied directly to our setting. To achieve our goal, we will follow the approach in $[\mathbf{2}],[\mathbf{3}],[\mathbf{1 8}]$, whose key ingredient is a sub-mean value property; see Lemma 3.4 below. This approach has recently been used in [16] to derive the equivalence of LittlewoodPaley $g$-function and area function characterizations of one-parameter Hardy spaces associated to operators. However, the Littlewood-Paley $g$-function and area function in 
[16] are only defined via the heat semigroup, which are less general than those defined in the current paper.

We close this introduction by making some conventions. Throughout this paper, we denote by $C$ and $c$ (possibly with subscripts) constants that may vary from place to place and may depend on any factor quantified (implicitly or explicitly) before its occurrence, but not on factors quantified afterwards. In some cases "sup" will mean "ess sup", which will be clear from the context.

\section{Preliminaries.}

In this section we collect some facts and technical results which will be needed in the subsequent section. We start by noting that, if $(X, \rho, \mu)$ is a space of homogeneous type, then for any $N>n$, there exists a constant $C=C(N)$ such that

$$
\int_{X}\left(1+\frac{\rho(x, y)}{t}\right)^{-N} d \mu(y) \leq C V(x, t)
$$

for all $x \in X$ and $t>0$.

The following lemma is essentially [4, Lemma 2.3]. See also [20, Lemma 2.1].

Lemma 2.1. Assume that $(X, \rho, \mu)$ is a space of homogeneous type and $L$ is a nonnegative self-adjoint operator on $L^{2}(X, d \mu)$ whose heat kernel obeys the Gaussian upper bound. Let $\Phi \in \mathcal{S}(\mathbb{R})$ be even functions. Then for every $N>0$, there exists a constant $C=C(\Phi, N)$ such that the kernel $K_{\Phi(t \sqrt{L})}(x, y)$ of the operator $\Phi(t \sqrt{L})$ satisfies

$$
\left|K_{\Phi(t \sqrt{L})}(x, y)\right| \leq \frac{C}{V(x, t)}\left(1+\frac{\rho(x, y)}{t}\right)^{-N}
$$

Lemma 2.2. Assume that $(X, \rho, \mu)$ is a space of homogeneous type and $L$ is a nonnegative self-adjoint operator on $L^{2}(X, d \mu)$ whose heat kernel obeys the Gaussian upper bound. Let $\Phi, \Psi \in \mathcal{S}(\mathbb{R})$ be even functions and let $\Psi$ satisfy

$$
\Psi^{(\nu)}(0)=0, \quad \nu=0,1, \ldots, m
$$

for some positive odd integer $m$. Then for every $N>0$, there exists a constant $C=$ $C(\Phi, \Psi, N, m)$ such that for all $s \geq t>0$,

$$
\left|K_{\Phi(s \sqrt{L}) \Psi(t \sqrt{L})}(x, y)\right| \leq C\left(\frac{t}{s}\right)^{m+1} \frac{1}{V(x, s)}\left(1+\frac{\rho(x, y)}{s}\right)^{-N} .
$$

Proof. First note that the property (2.2) implies that the function $\lambda \mapsto$ $\lambda^{-(m+1)} \Psi(\lambda)$ is an even function, smooth at 0 , and belongs to $\mathcal{S}(\mathbb{R})$. We set $\Phi_{m}(\lambda):=$ $\lambda^{m+1} \Phi(\lambda)$ and $\Psi_{m}(\lambda):=\lambda^{-(m+1)} \Psi(\lambda)$ for $\lambda \in \mathbb{R}$. Then both $\Phi_{m}$ and $\Psi_{m}$ are even functions and belong to $\mathcal{S}(\mathbb{R})$. Since

$$
\Phi(s \sqrt{L}) \Psi(t \sqrt{L})=\left(\frac{t}{s}\right)^{m+1}\left[(s \sqrt{L})^{m+1} \Phi(s \sqrt{L})\right]\left[(t \sqrt{L})^{-(m+1)} \Psi(t \sqrt{L})\right]
$$




$$
=\left(\frac{t}{s}\right)^{m+1} \Phi_{m}(s \sqrt{L}) \Psi_{m}(t \sqrt{L}),
$$

it follows from Lemma 2.1 that

$$
\begin{aligned}
& \left|K_{\Phi(s \sqrt{L}) \Psi(t \sqrt{L})}(x, y)\right| \\
& =\left(\frac{t}{s}\right)^{m+1}\left|K_{\Phi_{m}(s \sqrt{L}) \Psi_{m}(t \sqrt{L})}(x, y)\right| \\
& \leq\left(\frac{t}{s}\right)^{m+1} \int_{X}\left|K_{\Phi_{m}(s \sqrt{L})}(x, z) K_{\Psi_{m}(t \sqrt{L})}(z, y)\right| d \mu(z) \\
& \leq C(\Phi, \Psi, N, m)\left(\frac{t}{s}\right)^{m+1} \int_{X} \frac{1}{V(x, s)}\left(1+\frac{\rho(x, z)}{s}\right)^{-N} \\
& \quad \times \frac{1}{V(y, t)}\left(1+\frac{\rho(z, y)}{t}\right)^{-(N+n+1)} d \mu(z) .
\end{aligned}
$$

For $s \geq t>0$, we have

$$
\left(1+\frac{\rho(x, z)}{s}\right)^{-N}\left(1+\frac{\rho(z, y)}{t}\right)^{-N} \leq\left(1+\frac{\rho(x, y)}{s}\right)^{-N}
$$

This along with (2.1) yields

$$
\begin{aligned}
& \int_{X}\left(1+\frac{\rho(x, z)}{s}\right)^{-N}\left(1+\frac{\rho(z, y)}{t}\right)^{-(N+n+1)} d \mu(z) \\
& \leq\left(1+\frac{\rho(x, y)}{s}\right)^{-N} \int_{X}\left(1+\frac{\rho(z, y)}{t}\right)^{-(n+1)} d \mu(z) \\
& \leq C\left(1+\frac{\rho(x, y)}{s}\right)^{-N} V(y, t) .
\end{aligned}
$$

Combining (2.4) and (2.5) we obtain (2.3).

Lemma 2.3. Suppose $\Phi \in \mathcal{A}(\mathbb{R})$ is an even function. Then there exist even functions $\Psi, \Upsilon, \Theta \in \mathcal{S}(\mathbb{R})$ such that

$$
\begin{aligned}
& \operatorname{supp} \Upsilon \subset\{|\lambda| \leq 2 \varepsilon\}, \\
& \operatorname{supp} \Theta \subset\{\varepsilon / 2 \leq|\lambda| \leq 2 \varepsilon\}
\end{aligned}
$$

and

$$
\Psi(\lambda) \Upsilon(\lambda)+\sum_{k=1}^{\infty} \Phi\left(2^{-2 k} \lambda\right) \Theta\left(2^{-2 k} \lambda\right)=1 \quad \text { for all } \lambda \in \mathbb{R}
$$

where $\varepsilon$ is a constant from (1.4).

Proof. Define $\Psi(\lambda):=e^{-\lambda^{2}}, \lambda \in \mathbb{R}$. Obviously, $\Psi \in \mathcal{S}(\mathbb{R})$ and $\Psi$ is even. Choose 
nonnegative even functions $\Omega, \Gamma \in \mathcal{S}(\mathbb{R})$ such that

$$
\begin{aligned}
& \Omega(\lambda) \neq 0 \Longleftrightarrow|\lambda|<2 \varepsilon, \\
& \Gamma(\lambda) \neq 0 \Longleftrightarrow \varepsilon / 2<|\lambda|<2 \varepsilon .
\end{aligned}
$$

Then we set

$$
\Xi(\lambda):=\Psi(\lambda) \Omega(\lambda)+\sum_{k=1}^{\infty} \Phi\left(2^{-k} \lambda\right) \Gamma\left(2^{-k} \lambda\right), \quad \lambda \in \mathbb{R} .
$$

From the properties of $\Phi, \Psi, \Omega$ and $\Gamma$ it follows that $\Xi$ is strictly positive on $\mathbb{R}$. In addition, from the properties of $\Omega$ and $\Gamma$ we see that for any fixed $\lambda_{0} \in \mathbb{R} \backslash\{0\}$, the number of those $k$ 's for which $\Phi\left(2^{-k} \lambda\right) \Gamma\left(2^{-k} \lambda\right)$ do not vanish identically in $\left(4 \lambda_{0} / 5,6 \lambda_{0} / 5\right)$ is no more than 4 , which implies that $\Xi$ is smooth in $\left(4 \lambda_{0} / 5,6 \lambda_{0} / 5\right)$ and hence $\Xi \in C^{\infty}(\mathbb{R} \backslash\{0\})$. It is obvious that $\Xi$ is also smooth at the origin 0 . Therefore $\Xi \in C^{\infty}(\mathbb{R})$. Now define the functions $\Upsilon$ and $\Theta$ respectively by

$$
\Upsilon(\lambda):=\frac{\Omega(\lambda)}{\Xi(\lambda)} \text { and } \quad \Theta(\lambda):=\frac{\Gamma(\lambda)}{\Xi(\lambda)} .
$$

Then it is straightforward to verify that $\Psi, \Upsilon$ and $\Theta$ satisfy the desired properties.

The following lemma is a homogeneous analogy of Lemma 2.3. It can be obtained by slightly modifying the argument of Lemma 2.3 .

Lemma 2.4. Suppose $\Phi \in \mathcal{A}(\mathbb{R})$ is an even function. Then there exists an even function $\Theta \in \mathcal{S}(\mathbb{R})$ such that

$$
\operatorname{supp} \Theta \subset\{\varepsilon / 2 \leq|\lambda| \leq 2 \varepsilon\}
$$

and

$$
\sum_{k=-\infty}^{\infty} \Phi\left(2^{-k} \lambda\right) \Theta\left(2^{-k} \lambda\right)=1 \quad \text { for all } \lambda \in \mathbb{R} \backslash\{0\},
$$

where $\varepsilon$ is a constant from (1.4).

Lemma 2.5. Assume that $(X, \rho, \mu)$ is a space of homogeneous type with $\mu(X)=\infty$ and $L$ is a nonnegative self-adjoint operator on $L^{2}(X, d \mu)$ whose heat kernel obeys the Gaussian upper bound. Let $\{E(\lambda): \lambda \geq 0\}$ be spectral resolution of L. Then the spectral measure of the set $\{0\}$ is zero, i.e., the point $\lambda=0$ may be neglected in the spectral resolution.

Proof. Assume by contradiction that $E(\{0\}) \neq 0$, then there exists $g \in L^{2}(X)$ such that $f:=E(\{0\}) g$ is not the zero element in $L^{2}(X, d \mu)$. Since $E(\{0\})$ is a an orthogonal projection,

$$
E(\{0\}) f=E(\{0\}) E(\{0\}) g=E(\{0\}) g=f .
$$


It follows that for all $t>0$,

$$
e^{-t L} f=\int_{0}^{\infty} e^{-t \lambda} d E(\lambda) f=\int_{0}^{\infty} e^{-t \lambda} d E(\lambda) E(\{0\}) f=\int_{\{0\}} e^{-t \lambda} d E(\lambda) f=E(\{0\}) f=f .
$$

Hence, for a.e. $x \in X$ and all $t>0$, we have

$$
\begin{aligned}
|f(x)| & =\left|e^{-t L} f(x)\right| \leq \int_{X}\left|p_{t}(x, y) \| f(y)\right| d \mu(y) \\
& \leq\|f\|_{L^{2}(X, d \mu)}\left(\int_{X}\left|p_{t}(x, y)\right|^{2} d \mu(y)\right)^{1 / 2} \\
& \leq C\|f\|_{L^{2}(X, d \mu)}\left(\int_{X} \frac{1}{V(x, \sqrt{t})^{2}}\left(1+\frac{\rho(x, y)}{\sqrt{t}}\right)^{-(n+1)} d \mu(y)\right)^{1 / 2} \\
& \leq C\|f\|_{L^{2}(X, d \mu)} V(x, \sqrt{t})^{-1 / 2} .
\end{aligned}
$$

Since $\mu(X)=\infty$, letting $t \rightarrow \infty$ in the above inequalities yields that $f(x)=0$. Hence $f=0$ in $L^{2}(X, d \mu)$, which leads to a contradiction. Therefore we must have $E(\{0\})=0$.

The following two lemmas are two-parameter counterparts of Lemma 2 and Lemma 3 in [18], respectively. These can be proved by slightly modifying the proofs of the corresponding one-parameter results. We omit the details here.

Lemma 2.6 ([18, Lemma 2]). Let $0<p, q<\infty$ and $\sigma_{1}, \sigma_{2}>0$. Let $w$ be an arbitrary weight (i.e., nonnegative locally integrable function) on $X_{1} \times X_{2}$. Let $\left\{g_{j_{1}, j_{2}}\right\}_{j_{1}, j_{2}=-\infty}^{\infty}$ be a sequence of nonnegative measurable functions on $X_{1} \times X_{2}$ and put

$$
h_{j_{1}, j_{2}}\left(x_{1}, x_{2}\right)=\sum_{k_{1}=-\infty}^{\infty} \sum_{k_{2}=-\infty}^{\infty} 2^{-\left|k_{1}-j_{1}\right| \sigma_{1}} 2^{-\left|k_{2}-j_{2}\right| \sigma_{2}} g_{k_{1}, k_{2}}\left(x_{1}, x_{2}\right)
$$

for $\left(x_{1}, x_{2}\right) \in X_{1} \times X_{2}$ and $j_{1}, j_{2} \in \mathbb{Z}$. Then, there exists a constant $C=C\left(q, \sigma_{1}, \sigma_{2}\right)$ such that

$$
\left\|\left\{h_{j_{1}, j_{2}}\right\}_{j_{1}, j_{2}=-\infty}^{\infty}\right\|_{L_{w}^{p}\left(\ell^{q}\right)} \leq C\left\|\left\{g_{j_{1}, j_{2}}\right\}_{j_{1}, j_{2}=-\infty}^{\infty}\right\|_{L_{w}^{p}\left(\ell^{q}\right)}
$$

where

$$
\begin{aligned}
\left\|\left\{g_{j_{1}, j_{2}}\right\}_{j_{1}, j_{2}=-\infty}^{\infty}\right\|_{L_{w}^{p}\left(\ell^{q}\right)} & :=\|\left\{\left\{g_{j_{1}, j_{2}}\right\}_{j_{1}, j_{2}=-\infty}^{\infty}\left\|_{\ell_{q}}\right\|_{L_{w}^{p}\left(X_{1} \times X_{2}\right)}\right. \\
& =\left\|\left(\sum_{j_{1}=-\infty}^{\infty} \sum_{j_{2}=-\infty}^{\infty}\left|g_{j_{1}, j_{2}}\left(x_{1}, x_{2}\right)\right|^{q}\right)^{1 / q}\right\|_{L_{w}^{p}\left(X_{1} \times X_{2}\right)}
\end{aligned}
$$


Lemma 2.7 ([18, Lemma 3]). Let $0<r \leq 1$, and let $\left\{b_{j_{1}, j_{2}}\right\}_{j_{1}, j_{2}=-\infty}^{\infty}$ and $\left\{d_{j_{1}, j_{2}}\right\}_{j_{1}, j_{2}=-\infty}^{\infty}$ be two sequences taking values in $(0, \infty]$ and $(0, \infty)$ respectively. Assume that there exists $N_{0}>0$ such that

$$
d_{j_{1}, j_{2}}=O\left(2^{j_{1} N_{0}} 2^{j_{2} N_{0}}\right), \quad j_{1}, j_{2} \rightarrow \infty,
$$

and that for every $N>0$ there exists a finite constant $C=C_{N}$ such that

$$
d_{j_{1}, j_{2}} \leq C_{N} \sum_{k_{1}=j_{1}}^{\infty} \sum_{k_{2}=j_{2}}^{\infty} 2^{\left(j_{1}-k_{1}\right) N} 2^{\left(j_{2}-k_{2}\right) N} b_{k_{1}, k_{2}} d_{k_{1}, k_{2}}^{1-r}, \quad j_{1}, j_{2} \in \mathbb{Z} .
$$

Then for every $N>0$,

$$
d_{j_{1}, j_{2}}^{r} \leq C_{N} \sum_{k_{1}=j_{1}}^{\infty} \sum_{k_{2}=j_{2}}^{\infty} 2^{\left(j_{1}-k_{1}\right) N r} 2^{\left(j_{2}-k_{2}\right) N r} b_{k_{1}, k_{2}}, \quad j_{1}, j_{2} \in \mathbb{Z},
$$

with the same constants $C_{N}$.

For a locally integrable function $f$ on $X_{1} \times X_{2}$, the strong maximal function is defined by

$$
\mathcal{M}_{s}(f)\left(x_{1}, x_{2}\right):=\sup _{\left(x_{1}, x_{2}\right) \in B_{1} \times B_{2}} \frac{1}{\mu_{1}\left(B_{1}\right) \mu_{2}\left(B_{2}\right)} \iint_{B_{1} \times B_{2}}\left|f\left(y_{1}, y_{2}\right)\right| d \mu_{1}\left(y_{1}\right) d \mu_{2}\left(y_{2}\right),
$$

where $B_{i}$ runs over all balls in $X_{i}, i=1,2$. Using (1.3) and the volume doubling property, one can easily show that if $N_{i}>n_{i}+D_{i}$ for $i=1,2$, then

$$
\iint_{X_{1} \times X_{2}} \frac{\left|f\left(y_{1}, y_{2}\right)\right|}{\prod_{i=1}^{2} V\left(y_{i}, t_{i}\right)\left(1+t_{i}^{-1} \rho_{i}\left(x_{i}, y_{i}\right)\right)^{N_{i}}} d \mu_{1}\left(y_{1}\right) d \mu_{2}\left(y_{2}\right) \leq C \mathcal{M}_{s}(f)\left(x_{1}, x_{2}\right) .
$$

We will also need the following weighted vector-valued inequality for strong maximal functions on spaces of homogeneous type. See, for instance, [14] and [19].

Lemma 2.8. Suppose $1<p<\infty, 1<q \leq \infty$ and $w \in A_{p}\left(X_{1} \times X_{2}\right)$. Then there exists a constant $C$ such that

$$
\left\|\left\{\mathcal{M}_{s}\left(f_{j_{1}, j_{2}}\right)\right\}_{j_{1}, j_{2}=-\infty}^{\infty}\right\|_{L_{w}^{p}\left(\ell^{q}\right)} \leq C\left\|\left\{f_{j_{1}, j_{2}}\right\}_{j_{1}, j_{2}=-\infty}^{\infty}\right\|_{L_{w}^{p}\left(\ell^{q}\right)}
$$

for all sequences $\left\{f_{j_{1}, j_{2}}\right\}_{j_{1}, j_{2}=-\infty}^{\infty}$ on $X_{1} \times X_{2}$, where the space $L_{w}^{p}\left(\ell^{q}\right)$ is defined by $(2.6)$.

\section{Proofs of Theorems 1.4 and $\mathbf{1 . 5}$.}

We divide the proofs of Theorems 1.4 and 1.5 into a sequence of lemmas.

The following lemma is standard; see, for instance, [22, Theorem 4 in Chapter 4].

Lemma 3.1. Let $\Phi_{1}, \Phi_{2} \in \mathcal{S}(\mathbb{R})$ be even functions. Let $p \in(0, \infty), w \in A_{\infty}\left(X_{1} \times\right.$ $\left.X_{2}\right)$, and $\lambda_{1}, \lambda_{2}>2 q_{w} / \min \{p, 2\}$. Then there exists a constant $C$ such that for all $f \in L^{2}\left(X_{1} \times X_{2}\right)$, 


$$
\left\|g_{\Phi_{1}, \Phi_{2}, L_{1}, L_{2}, \lambda_{1}, \lambda_{2}}^{*}(f)\right\|_{L_{w}^{p}\left(X_{1} \times X_{2}\right)} \leq C\left\|S_{\Phi_{1}, \Phi_{2}, L_{1}, L_{2}}(f)\right\|_{L_{w}^{p}\left(X_{1} \times X_{2}\right)} .
$$

Lemma 3.2. Let $\Phi_{1}, \Phi_{2} \in \mathcal{S}(\mathbb{R})$ be even functions. Let $p \in(0, \infty), \lambda_{1}, \lambda_{2}>0$, and $w$ be an arbitrary weight (i.e., nonnegative locally integrable function) on $X_{1} \times X_{2}$. Then there exists a constant $C$ such that for all $f \in L^{2}\left(X_{1} \times X_{2}\right)$,

$$
\begin{aligned}
& \left\|S_{\Phi_{1}, \Phi_{2}, L_{1}, L_{2}}(f)\right\|_{L_{w}^{p}\left(X_{1} \times X_{2}\right)} \\
& \quad \leq C\left\|\left(\int_{0}^{\infty} \int_{0}^{\infty}\left|\left[\Phi_{1}\left(t_{1} \sqrt{L_{1}}\right) \otimes \Phi_{2}\left(t_{2} \sqrt{L_{2}}\right)\right]_{\lambda_{1}, \lambda_{2}}^{*} f\right|^{2} \frac{d t_{1}}{t_{1}} \frac{d t_{2}}{t_{2}}\right)^{1 / 2}\right\|_{L_{w}^{p}\left(X_{1} \times X_{2}\right)} .
\end{aligned}
$$

Proof. Observe that for all $\lambda_{1}, \lambda_{2}, t_{1}, t_{2}>0$ and all $\left(x_{1}, x_{2}\right) \in X_{1} \times X_{2}$,

$$
\begin{gathered}
\frac{1}{V\left(x_{1}, t_{1}\right) V\left(x_{2}, t_{2}\right)} \iint_{B\left(x_{1}, t_{1}\right) \times B\left(x_{2}, t_{2}\right)}\left|\Phi_{1}\left(t_{1} \sqrt{L_{1}}\right) \otimes \Phi_{2}\left(t_{2} \sqrt{L_{2}}\right) f\left(y_{1}, y_{2}\right)\right|^{2} d \mu_{1}\left(y_{1}\right) d \mu_{2}\left(y_{2}\right) \\
\leq \sup _{\left(y_{1}, y_{2}\right) \in B\left(x_{1}, t_{1}\right) \times B\left(x_{2}, t_{2}\right)}\left|\Phi_{1}\left(t_{1} \sqrt{L_{1}}\right) \otimes \Phi_{2}\left(t_{2} \sqrt{L_{2}}\right) f\left(y_{1}, y_{2}\right)\right|^{2} \\
\leq 2^{2 \lambda_{1}} 2^{2 \lambda_{2}}\left|\left[\Phi_{1}\left(t_{1} \sqrt{L_{1}}\right) \otimes \Phi_{2}\left(t_{2} \sqrt{L_{2}}\right)\right]_{\lambda_{1}, \lambda_{2}}^{*} f\left(x_{1}, x_{2}\right)\right|^{2} .
\end{gathered}
$$

Taking the norm $\int_{0}^{\infty} \int_{0}^{\infty}|\cdot|\left(d t_{1} / t_{1}\right)\left(d t_{2} / t_{2}\right)$ on both sides gives the pointwise estimate

$$
\begin{aligned}
& {\left[S_{\Phi_{1}, \Phi_{2}, L_{1}, L_{2}}(f)\left(x_{1}, x_{2}\right)\right]^{2}} \\
& \quad \leq 2^{2 \lambda_{1}} 2^{2 \lambda_{2}} \int_{0}^{\infty} \int_{0}^{\infty}\left|\left[\Phi_{1}\left(t_{1} \sqrt{L_{1}}\right) \otimes \Phi_{2}\left(t_{2} \sqrt{L_{2}}\right)\right]_{\lambda_{1}, \lambda_{2}}^{*} f\left(x_{1}, x_{2}\right)\right|^{2} \frac{d t_{1}}{t_{1}} \frac{d t_{2}}{t_{2}},
\end{aligned}
$$

which readily yields the desired estimate.

Lemma 3.3. Suppose $\Phi_{1}, \Phi_{2}, \widetilde{\Phi}_{1}, \widetilde{\Phi}_{2} \in \mathcal{A}(\mathbb{R})$ are even functions satisfying

$$
\Phi_{1}(0)=\Phi_{2}(0)=\widetilde{\Phi}_{1}(0)=\widetilde{\Phi}_{2}(0)=0 .
$$

Let $p \in(0, \infty), \lambda_{1}, \lambda_{2}>0$, and $w$ be an arbitrary weight (i.e., nonnegative locally integrable function) on $X_{1} \times X_{2}$. Then there exists a constant $C$ such that for all $f \in$ $L^{2}\left(X_{1} \times X_{2}\right)$,

$$
\begin{aligned}
& \left\|\left(\int_{0}^{\infty} \int_{0}^{\infty}\left|\left[\widetilde{\Phi}_{1}\left(t_{1} \sqrt{L_{1}}\right) \otimes \widetilde{\Phi}_{2}\left(t_{2} \sqrt{L_{2}}\right)\right]_{\lambda_{1}, \lambda_{2}}^{*} f\right|^{2} \frac{d t_{1}}{t_{1}} \frac{d t_{2}}{t_{2}}\right)^{1 / 2}\right\|_{L_{w}^{p}\left(X_{1} \times X_{2}\right)} \\
& \quad \sim\left\|\left(\int_{0}^{\infty} \int_{0}^{\infty}\left|\left[\Phi_{1}\left(t_{1} \sqrt{L_{1}}\right) \otimes \Phi_{2}\left(t_{2} \sqrt{L_{2}}\right)\right]_{\lambda_{1}, \lambda_{2}}^{*} f\right|^{2} \frac{d t_{1}}{t_{1}} \frac{d t_{2}}{t_{2}}\right)^{1 / 2}\right\|_{L_{w}^{p}\left(X_{1} \times X_{2}\right)} .
\end{aligned}
$$

Proof. For $i=1,2$, since $\Phi_{i} \in \mathcal{A}(\mathbb{R})$ and $\Phi_{i}$ is even, by Lemma 2.4 there exists an even function $\Theta_{i} \in \mathcal{S}(\mathbb{R})$ such that $\operatorname{supp} \Theta_{i} \subset\left\{\varepsilon_{i} / 2 \leq|\lambda| \leq 2 \varepsilon_{i}\right\}$ and

$$
\sum_{k=-\infty}^{\infty} \Phi_{i}\left(2^{-k} \lambda\right) \Theta_{i}\left(2^{-k} \lambda\right)=1 \quad \text { for } \lambda \in \mathbb{R} \backslash\{0\},
$$


where $\varepsilon_{i}$ is the constant in the Tauberian condition (1.4) corresponding to $\Phi_{i}$. Hence it follows from Lemma 2.5 and the spectral theorem that for all $f \in L^{2}\left(X_{1} \times X_{2}\right)$ and $t_{1}, t_{2} \in[1,2]$,

$f=\sum_{k_{1}=-\infty}^{\infty} \sum_{k_{2}=-\infty}^{\infty}\left(\Phi_{1}\left(2^{-k_{1}} t_{1} \sqrt{L_{1}}\right) \Theta_{1}\left(2^{-k_{1}} t_{1} \sqrt{L_{1}}\right)\right) \otimes\left(\Phi_{2}\left(2^{-k_{2}} t_{2} \sqrt{L_{2}}\right) \Theta_{2}\left(2^{-k_{2}} t_{2} \sqrt{L_{2}}\right)\right) f$

with convergence in the sense of $L^{2}\left(X_{1} \times X_{2}\right)$ norm. Consequently, for all $j_{1}, j_{2} \in \mathbb{Z}$, all $t_{1}, t_{2} \in[1,2]$ and a.e. $\left(y_{1}, y_{2}\right) \in X_{1} \times X_{2}$,

$$
\begin{aligned}
\widetilde{\Phi}_{1}\left(2^{-j_{1}} t_{1} \sqrt{L_{1}}\right) \otimes \widetilde{\Phi}_{2}\left(2^{-j_{2}} t_{2} \sqrt{L_{2}}\right) f\left(y_{1}, y_{2}\right) \\
=\sum_{k_{1}=-\infty}^{\infty} \sum_{k_{2}=-\infty}^{\infty}\left(\widetilde{\Phi}_{1}\left(2^{-j_{1}} t_{1} \sqrt{L_{1}}\right) \Phi_{1}\left(2^{-k_{1}} t_{1} \sqrt{L_{1}}\right) \Theta_{1}\left(2^{-k_{1}} t_{1} \sqrt{L_{1}}\right)\right) \\
\otimes\left(\widetilde{\Phi}_{2}\left(2^{-j_{2}} t_{2} \sqrt{L_{2}}\right) \Phi_{2}\left(2^{-k_{2}} t_{2} \sqrt{L_{2}}\right) \Theta_{2}\left(2^{-k_{2}} t_{2} \sqrt{L_{2}}\right)\right) f\left(y_{1}, y_{2}\right) \\
=\sum_{k_{1}=-\infty}^{\infty} \sum_{k_{2}=-\infty}^{\infty} \iint_{X_{1} \times X_{2}} K_{\widetilde{\Phi}_{1}\left(2^{-j_{1}} t_{1} \sqrt{L_{1}}\right) \Theta_{1}\left(2^{-k_{1}} t_{1} \sqrt{L_{1}}\right)}\left(y_{1}, z_{1}\right) \\
\quad \times K_{\widetilde{\Phi}_{2}\left(2^{-j_{2}} t_{2} \sqrt{L_{2}}\right) \Theta_{2}\left(2^{-k_{2}} t_{2} \sqrt{L_{2}}\right)}\left(y_{2}, z_{2}\right) \\
\quad \times\left(\Phi_{1}\left(2^{-k_{1}} t_{1} \sqrt{L_{1}}\right) \otimes \Phi_{2}\left(2^{-k_{2}} t_{2} \sqrt{L_{2}}\right)\right) f\left(z_{1}, z_{2}\right) d \mu_{1}\left(z_{1}\right) d \mu_{2}\left(z_{2}\right) .
\end{aligned}
$$

Since $\widetilde{\Phi}_{i}$ is even on $\mathbb{R}$, we have $\widetilde{\Phi}_{i}^{\prime}(0)=0$, for $i=1,2$. Thus $\widetilde{\Phi}_{i}(0)=\widetilde{\Phi}_{i}^{\prime}(0)=0$ for $i=1,2$. On the other hand, since $\Theta_{i}$ vanishes near the origin, we have $\Theta_{i}^{(\nu)}(0)=0$ for every non-negative integer $\nu$. Hence it follows from Lemma 2.2 that for any positive integer $m$ and any $N>0$,

$$
\begin{aligned}
& \left|K_{\widetilde{\Phi}_{i}\left(2^{-j_{i}} t_{i} \sqrt{L_{i}}\right) \Theta_{i}\left(2^{-k_{i}} t_{i} \sqrt{L_{i}}\right)}\left(y_{i}, z_{i}\right)\right| \\
& \quad \leq \begin{cases}C\left(\widetilde{\Phi}_{i}, \Theta_{i}, N\right) 2^{-2\left|j_{i}-k_{i}\right|} V\left(y_{i}, 2^{-k_{i}} t_{i}\right)^{-1}\left(1+2^{k_{i}} t_{i}^{-1} \rho_{i}\left(y_{i}, z_{i}\right)\right)^{-N}, & j_{i} \geq k_{i}, \\
C\left(\widetilde{\Phi}_{i}, \Theta_{i}, N, m\right) 2^{-m\left|j_{i}-k_{i}\right|} V\left(y_{i}, 2^{-j_{i}} t_{i}\right)^{-1}\left(1+2^{j_{i}} t_{i}^{-1} \rho_{i}\left(y_{i}, z_{i}\right)\right)^{-N}, & j_{i}<k_{i} .\end{cases}
\end{aligned}
$$

Choose $N \geq \max \left\{\lambda_{1}+n_{1}+1, \lambda_{2}+n_{2}+1\right\}$, then from (3.1), (3.2) and the inequality

$$
\begin{aligned}
\mid( & \left.\Phi_{1}\left(2^{-k_{1}} t_{1} \sqrt{L_{1}}\right) \otimes \Phi_{2}\left(2^{-k_{2}} t_{2} \sqrt{L_{2}}\right)\right) f\left(z_{1}, z_{2}\right) \mid \\
\leq & {\left[\Phi_{1}\left(2^{-k_{1}} t_{1} \sqrt{L_{1}}\right) \otimes \Phi_{2}\left(2^{-k_{2}} t_{2} \sqrt{L_{2}}\right)\right]_{\lambda_{1}, \lambda_{2}}^{*} f\left(x_{1}, x_{2}\right) } \\
\quad & \times\left(1+2^{k_{1}} t_{1}^{-1} \rho_{1}\left(x_{1}, z_{1}\right)\right)^{\lambda_{1}}\left(1+2^{k_{2}} t_{2}^{-1} \rho_{2}\left(x_{2}, z_{2}\right)\right)^{\lambda_{2}},
\end{aligned}
$$

we infer that

$$
\begin{aligned}
& {\left[\widetilde{\Phi}_{1}\left(2^{-j_{1}} t_{1} \sqrt{L_{1}}\right) \otimes \widetilde{\Phi}_{2}\left(2^{-j_{2}} t_{2} \sqrt{L_{2}}\right)\right]_{\lambda_{1}, \lambda_{2}}^{*} f\left(x_{1}, x_{2}\right)} \\
& \quad \leq \sum_{k_{1}=-\infty}^{\infty} \sum_{k_{2}=-\infty}^{\infty} \gamma_{j_{1}, k_{1}, j_{2}, k_{2}}\left[\Phi_{1}\left(2^{-k_{1}} t_{1} \sqrt{L_{1}}\right) \otimes \Phi_{2}\left(2^{-k_{2}} t_{2} \sqrt{L_{2}}\right)\right]_{\lambda_{1}, \lambda_{2}}^{*} f\left(x_{1}, x_{2}\right)
\end{aligned}
$$




$$
\begin{gathered}
\times \sup _{\left(y_{1}, y_{2}\right) \in X_{1} \times X_{2}} \iint_{X_{1} \times X_{2}} \frac{\left(1+2^{k_{i}} t_{i}^{-1} \rho_{i}\left(x_{i}, z_{i}\right)\right)^{\lambda_{i}}}{\prod_{i=1}^{2}\left(1+2^{j_{i}} t_{i}^{-1} \rho_{i}\left(x_{i}, y_{i}\right)\right)^{\lambda_{i}}\left(1+2^{j_{i} \wedge k_{i}} t_{i}^{-1} \rho_{i}\left(y_{i}, z_{i}\right)\right)^{\lambda_{i}+n_{i}+1}} \\
\times \frac{d \mu_{1}\left(z_{1}\right) d \mu_{2}\left(z_{2}\right)}{V\left(y_{1}, 2^{-\left(j_{1} \wedge k_{1}\right)} t_{1}\right) V\left(y_{2}, 2^{-\left(j_{2} \wedge k_{2}\right)} t_{2}\right)}
\end{gathered}
$$

where $j_{i} \wedge k_{i}:=\min \left\{j_{i}, k_{i}\right\}$ and

$$
\gamma_{j_{1}, k_{1}, j_{2}, k_{2}}:= \begin{cases}2^{-2\left|j_{1}-k_{1}\right|} 2^{-2\left|j_{2}-k_{2}\right|} & \text { if } j_{1} \geq k_{1} \text { and } j_{2} \geq k_{2}, \\ 2^{-2\left|j_{1}-k_{1}\right|} 2^{-m\left|j_{2}-k_{2}\right|} & \text { if } j_{1} \geq k_{1} \text { and } j_{2}<k_{2}, \\ 2^{-m\left|j_{1}-k_{1}\right|} 2^{-2\left|j_{2}-k_{2}\right|} & \text { if } j_{1}<k_{1} \text { and } j_{2} \geq k_{2}, \\ 2^{-m\left|j_{1}-k_{1}\right|} 2^{-m\left|j_{2}-k_{2}\right|} & \text { if } j_{1} \geq k_{1} \text { and } j_{2} \geq k_{2} .\end{cases}
$$

Using (2.1) and the fundamental inequality

$$
\begin{aligned}
(1 & \left.+2^{k_{i}} t_{i}^{-1} \rho_{i}\left(x_{i}, z_{i}\right)\right)^{\lambda_{i}} \\
& \leq \begin{cases}\left(1+2^{j_{i}} t_{i}^{-1} \rho_{i}\left(x_{i}, y_{i}\right)\right)^{\lambda_{i}}\left(1+2^{k_{i}} t_{i}^{-1} \rho_{i}\left(y_{i}, z_{i}\right)\right)^{\lambda_{i}}, & j_{i} \geq k_{i}, \\
2^{\left(k_{i}-j_{i}\right) \lambda_{i}}\left(1+2^{j_{i}} t_{i}^{-1} \rho_{i}\left(x_{i}, y_{i}\right)\right)^{\lambda_{i}}\left(1+2^{j_{i}} t_{i}^{-1} \rho_{i}\left(y_{i}, z_{i}\right)\right)^{\lambda_{i}}, & j_{i}<k_{i},\end{cases}
\end{aligned}
$$

it follows that

$$
\begin{aligned}
& {\left[\widetilde{\Phi}_{1}\left(2^{-j_{1}} t_{1} \sqrt{L_{1}}\right) \otimes \widetilde{\Phi}_{2}\left(2^{-j_{2}} t_{2} \sqrt{L_{2}}\right)\right]_{\lambda_{1}, \lambda_{2}}^{*} f\left(x_{1}, x_{2}\right)} \\
& \quad \leq \sum_{k_{1}=-\infty}^{\infty} \sum_{k_{2}=-\infty}^{\infty} \gamma_{j_{1}, k_{1}, j_{2}, k_{2}}^{\prime}\left[\Phi_{1}\left(2^{-k_{1}} t_{1} \sqrt{L_{1}}\right) \otimes \Phi_{2}\left(2^{-k_{2}} t_{2} \sqrt{L_{2}}\right)\right]_{\lambda_{1}, \lambda_{2}}^{*} f\left(x_{1}, x_{2}\right)
\end{aligned}
$$

where

$$
\gamma_{j_{1}, k_{1}, j_{2}, k_{2}}^{\prime}:= \begin{cases}2^{-2\left|j_{1}-k_{1}\right|} 2^{-2\left|j_{2}-k_{2}\right|} & \text { if } j_{1} \geq k_{1} \text { and } j_{2} \geq k_{2}, \\ 2^{-2\left|j_{1}-k_{1}\right|} 2^{-\left(m-\lambda_{2}\right)\left|j_{2}-k_{2}\right|} & \text { if } j_{1} \geq k_{1} \text { and } j_{2}<k_{2}, \\ 2^{-\left(m-\lambda_{1}\right)\left|j_{1}-k_{1}\right|} 2^{-2\left|j_{2}-k_{2}\right|} & \text { if } j_{1}<k_{1} \text { and } j_{2} \geq k_{2}, \\ 2^{-\left(m-\lambda_{1}\right)\left|j_{1}-k_{1}\right|} 2^{-\left(m-\lambda_{2}\right)\left|j_{2}-k_{2}\right|} & \text { if } j_{1} \geq k_{1} \text { and } j_{2} \geq k_{2} .\end{cases}
$$

Now let us choose $m>\max \left\{\lambda_{1}, \lambda_{2}\right\}$ and set $\sigma:=\min \left\{m-\lambda_{1}, m-\lambda_{2}, 2\right\}$. Then (3.3) implies that

$$
\begin{aligned}
& {\left[\widetilde{\Phi}_{1}\left(2^{-j_{1}} t_{1} \sqrt{L_{1}}\right) \otimes \widetilde{\Phi}_{2}\left(2^{-j_{2}} t_{2} \sqrt{L_{2}}\right)\right]_{\lambda_{1}, \lambda_{2}}^{*} f\left(x_{1}, x_{2}\right)} \\
& \leq \sum_{k_{1}=-\infty}^{\infty} \sum_{k_{2}=-\infty}^{\infty} 2^{-\left|j_{1}-k_{1}\right| \sigma} 2^{-\left|j_{2}-k_{2}\right| \sigma}\left[\widetilde{\Phi}_{1}\left(2^{-k_{1}} t_{1} \sqrt{L_{1}}\right) \otimes \widetilde{\Phi}_{2}\left(2^{-k_{2}} t_{2} \sqrt{L_{2}}\right)\right]_{\lambda_{1}, \lambda_{2}}^{*} f\left(x_{1}, x_{2}\right) .
\end{aligned}
$$

Taking on both sides the norm $\left(\int_{1}^{2} \int_{1}^{2}|\cdot|^{2}\left(d t_{1} / t_{1}\right)\left(d t_{2} / t_{2}\right)\right)^{1 / 2}$ and using Minkowski's inequality, we get

$$
\left(\int_{1}^{2} \int_{1}^{2}\left\{\left[\widetilde{\Phi}_{1}\left(2^{-j_{1}} t_{1} \sqrt{L_{1}}\right) \otimes \widetilde{\Phi}_{2}\left(2^{-j_{2}} t_{2} \sqrt{L_{2}}\right)\right]_{\lambda_{1}, \lambda_{2}}^{*} f\left(x_{1}, x_{2}\right)\right\}^{2} \frac{d t_{1}}{t_{1}} \frac{d t_{2}}{t_{2}}\right)^{1 / 2}
$$




$$
\begin{aligned}
\leq & C \sum_{k_{1}=-\infty}^{\infty} \sum_{k_{2}=-\infty}^{\infty} 2^{-\left|j_{1}-k_{1}\right| \sigma} 2^{-\left|j_{2}-k_{2}\right| \sigma} \\
& \times\left(\int_{1}^{2} \int_{1}^{2}\left\{\left[\Phi_{1}\left(2^{-k_{1}} t_{1} \sqrt{L_{1}}\right) \otimes \Phi_{2}\left(2^{-j_{2}} t_{2} \sqrt{L_{2}}\right)\right]_{\lambda_{1}, \lambda_{2}}^{*} f\left(x_{1}, x_{2}\right)\right\}^{2} \frac{d t_{1}}{t_{1}} \frac{d t_{2}}{t_{2}}\right)^{1 / 2} .
\end{aligned}
$$

Finally, applying Lemma 2.6 in $L^{p}\left(\ell^{2}\right)$ yields

$$
\begin{aligned}
& \left\|\left(\int_{0}^{\infty} \int_{0}^{\infty}\left|\left[\widetilde{\Phi}_{1}\left(t_{1} \sqrt{L_{1}}\right) \otimes \widetilde{\Phi}_{2}\left(t_{2} \sqrt{L_{2}}\right)\right]_{\lambda_{1}, \lambda_{2}}^{*} f\right|^{2} \frac{d t_{1}}{t_{1}} \frac{d t_{2}}{t_{2}}\right)^{1 / 2}\right\|_{L_{w}^{p}\left(X_{1} \times X_{2}\right)} \\
& \quad \leq C\left\|\left(\int_{0}^{\infty} \int_{0}^{\infty}\left|\left[\Phi_{1}\left(t_{1} \sqrt{L_{1}}\right) \otimes \Phi_{2}\left(t_{2} \sqrt{L_{2}}\right)\right]_{\lambda_{1}, \lambda_{2}}^{*} f\right|^{2} \frac{d t_{1}}{t_{1}} \frac{d t_{2}}{t_{2}}\right)^{1 / 2}\right\|_{L_{w}^{p}\left(X_{1} \times X_{2}\right)} .
\end{aligned}
$$

By symmetry, the converse inequality of (3.4) also holds. The proof of the lemma is complete.

Lemma 3.4. Let $\Phi_{1}, \Phi_{2} \in \mathcal{A}(\mathbb{R})$ be even functions. Then for any $r>0, \sigma>0$, $\lambda_{1}>D_{1} / 2$ and $\lambda_{2}>D_{2} / 2$, there exists a constant $C$ such that for all $f \in L^{2}\left(X_{1} \times X_{2}\right)$, all $\left(x_{1}, x_{2}\right) \in X_{1} \times X_{2}$ and all $t_{1}, t_{2} \in[1,2]$,

$$
\begin{aligned}
& \left\{\left[\Phi_{1}\left(2^{-j_{1}} t_{1} \sqrt{L_{1}}\right) \otimes \Phi_{2}\left(2^{-j_{2}} t_{2} \sqrt{L_{2}}\right)\right]_{\lambda_{1}, \lambda_{2}}^{*} f\left(x_{1}, x_{2}\right)\right\}^{r} \\
& \leq C \sum_{k_{1}=j_{1}}^{\infty} \sum_{k_{2}=j_{2}}^{\infty} 2^{\left(j_{1}-k_{1}\right) \sigma} 2^{\left(j_{2}-k_{2}\right) \sigma} \iint_{X_{1} \times X_{2}} \\
& \quad \times \frac{\left|\Phi_{1}\left(2^{-k_{1}} t_{1} \sqrt{L_{1}}\right) \otimes \Phi_{2}\left(2^{-k_{2}} t_{2} \sqrt{L_{2}}\right) f\left(z_{1}, z_{2}\right)\right|^{r} d \mu_{1}\left(z_{1}\right) d \mu_{2}\left(z_{2}\right)}{V\left(z_{1}, 2^{-k_{1}} t_{1}\right)\left(1+2^{k_{1}} t_{1}^{-1} \rho\left(x_{1}, z_{1}\right)\right)^{\lambda_{1} r} V\left(z_{2}, 2^{-k_{2}} t_{2}\right)\left(1+2^{k_{2}} t_{2}^{-1} \rho_{2}\left(x_{2}, z_{2}\right)\right)^{\lambda_{2} r}} .
\end{aligned}
$$

Proof. By Lemma 2.3, for $i=1,2$ there exist even functions $\Psi_{i}, \Upsilon_{i}, \Theta_{i} \in \mathcal{S}(\mathbb{R})$ such that $\operatorname{supp} \Upsilon_{i} \subset\left\{|\lambda| \leq 2 \varepsilon_{i}\right\}$, supp $\Theta_{i} \subset\left\{\varepsilon_{i} / 2 \leq|\lambda| \leq 2 \varepsilon_{i}\right\}$, and

$$
\Psi_{i}(\lambda) \Upsilon_{i}(\lambda)+\sum_{k_{i}=1}^{\infty} \Phi_{i}\left(2^{-k_{i}} \lambda\right) \Theta_{i}\left(2^{-k_{i}} \lambda\right)=1 \quad \text { for all } \lambda \in \mathbb{R}
$$

where $\varepsilon_{i}$ is the constant in the Tauberian condition (1.4) corresponding to $\Phi_{i}$. Replacing $\lambda$ with $2^{-j_{i}} t_{i} \lambda$ in (3.6), we see that for all $j_{i} \in \mathbb{Z}$ and $t_{i} \in[1,2]$,

$$
\Psi_{i}\left(2^{-j_{i}} t_{i} \lambda\right) \Upsilon_{i}\left(2^{-j_{i}} t_{i} \lambda\right)+\sum_{k_{i}=1}^{\infty} \Phi_{i}\left(2^{-\left(k_{i}+j_{i}\right)} t_{i} \lambda\right) \Theta_{i}\left(2^{-\left(k_{i}+j_{i}\right)} t_{i} \lambda\right)=1 .
$$

It then follows from the spectral theorem that for all $f \in L^{2}\left(X_{1} \times X_{2}\right)$, all $j_{1}, j_{2} \in \mathbb{Z}$ and all $t_{1}, t_{2} \in[1,2]$,

$$
f=\left(\Psi_{1}\left(2^{-j_{1}} t_{1} \sqrt{L_{1}}\right) \Upsilon_{1}\left(2^{-j_{1}} t_{1} \sqrt{L_{1}}\right)\right) \otimes\left(\Psi_{2}\left(2^{-j_{2}} t_{2} \sqrt{L_{2}}\right) \Upsilon_{2}\left(2^{-j_{2}} t_{2} \sqrt{L_{2}}\right)\right) f
$$




$$
\begin{gathered}
+\sum_{k_{1}=1}^{\infty}\left(\Phi_{1}\left(2^{-\left(k_{1}+j_{1}\right)} t_{1} \sqrt{L_{1}}\right) \Theta_{1}\left(2^{-\left(k_{1}+j_{1}\right)} t_{1} \sqrt{L_{1}}\right)\right) \\
\otimes\left(\Psi_{2}\left(2^{-j_{2}} t_{2} \sqrt{L_{2}}\right) \Upsilon_{2}\left(2^{-j_{2}} t_{2} \sqrt{L_{2}}\right)\right) f \\
+\sum_{k_{2}=1}^{\infty}\left(\Psi_{1}\left(2^{-j_{1}} t_{1} \sqrt{L_{1}}\right) \Upsilon_{1}\left(2^{-j_{1}} t_{1} \sqrt{L_{1}}\right)\right) \\
+\left(\Phi_{2}\left(2^{-\left(k_{2}+j_{2}\right)} t_{2} \sqrt{L_{2}}\right) \Theta_{2}\left(2^{-\left(k_{2}+j_{2}\right)} t_{2} \sqrt{L_{2}}\right)\right) f \\
+\sum_{k_{1}=1}^{\infty} \sum_{k_{2}=1}^{\infty}\left(\Phi_{1}\left(2^{-\left(k_{1}+j_{1}\right)} t_{1} \sqrt{L_{1}}\right) \Theta_{1}\left(2^{-\left(k_{1}+j_{1}\right)} t_{1} \sqrt{L_{1}}\right)\right) \\
\otimes\left(\Phi_{2}\left(2^{-\left(k_{2}+j_{2}\right)} t_{2} \sqrt{L_{2}}\right) \Theta_{2}\left(2^{-\left(k_{2}+j_{2}\right)} t_{2} \sqrt{L_{2}}\right)\right) f
\end{gathered}
$$

with convergence in the sense of $L^{2}\left(X_{1} \times X_{2}\right)$ norm. Hence, for all $j_{1}, j_{2} \in \mathbb{Z}$ and a.e. $\left(y_{1}, y_{2}\right) \in X_{1} \times X_{2}$, we have

$$
\begin{aligned}
& \Phi_{1}\left(2^{-j_{1}} t_{1} \sqrt{L_{1}}\right) \otimes \Phi_{2}\left(2^{-j_{2}} t_{2} \sqrt{L_{2}}\right) f\left(y_{1}, y_{2}\right) \\
& =\left(\Phi_{1}\left(2^{-j_{1}} t_{1} \sqrt{L_{1}}\right) \Psi_{1}\left(2^{-j_{1}} t_{1} \sqrt{L_{1}}\right) \Upsilon_{1}\left(2^{-j_{1}} t_{1} \sqrt{L_{1}}\right)\right) \\
& \otimes\left(\Phi_{2}\left(2^{-j_{2}} t_{2} \sqrt{L_{2}}\right) \Psi_{2}\left(2^{-j_{2}} t_{2} \sqrt{L_{2}}\right) \Upsilon_{2}\left(2^{-j_{2}} t_{2} \sqrt{L_{2}}\right)\right) f\left(y_{1}, y_{2}\right) \\
& +\sum_{k_{1}=1}^{\infty}\left(\Phi_{1}\left(2^{-j_{1}} t_{1} \sqrt{L_{1}}\right) \Phi_{1}\left(2^{-\left(k_{1}+j_{1}\right)} t_{1} \sqrt{L_{1}}\right) \Theta_{1}\left(2^{-\left(k_{1}+j_{1}\right)} t_{1} \sqrt{L_{1}}\right)\right) \\
& \otimes\left(\Phi_{2}\left(2^{-j_{2}} t_{2} \sqrt{L_{2}}\right) \Psi_{2}\left(2^{-j_{2}} t_{2} \sqrt{L_{2}}\right) \Upsilon_{2}\left(2^{-j_{2}} t_{2} \sqrt{L_{2}}\right)\right) f\left(y_{1}, y_{2}\right) \\
& +\sum_{k_{2}=1}^{\infty}\left(\Phi_{1}\left(2^{-j_{1}} t_{1} \sqrt{L_{1}}\right) \Psi_{1}\left(2^{-j_{1}} t_{1} \sqrt{L_{1}}\right) \Upsilon_{1}\left(2^{-j_{1}} t_{1} \sqrt{L_{1}}\right)\right) \\
& \otimes\left(\Phi_{2}\left(2^{-j_{2}} t_{2} \sqrt{L_{2}}\right) \Phi_{2}\left(2^{-\left(k_{2}+j_{2}\right)} t_{2} \sqrt{L_{2}}\right) \Theta_{2}\left(2^{-\left(k_{2}+j_{2}\right)} t_{2} \sqrt{L_{2}}\right)\right) f \\
& +\sum_{k_{1}=1}^{\infty} \sum_{k_{2}=1}^{\infty}\left(\Phi_{1}\left(2^{-j_{1}} t_{1} \sqrt{L_{1}}\right) \Phi_{1}\left(2^{-\left(k_{1}+j_{1}\right)} t_{1} \sqrt{L_{1}}\right) \Theta_{1}\left(2^{-\left(k_{1}+j_{1}\right)} t_{1} \sqrt{L_{1}}\right)\right) \\
& \otimes\left(\Phi_{2}\left(2^{-j_{2}} t_{2} \sqrt{L_{2}}\right) \Phi_{2}\left(2^{-\left(k_{2}+j_{2}\right)} t_{2} \sqrt{L_{2}}\right) \Theta_{2}\left(2^{-\left(k_{2}+j_{2}\right)} t_{2} \sqrt{L_{2}}\right)\right) f\left(y_{1}, y_{2}\right) \\
& =\iint_{X_{1} \times X_{2}} K_{\Psi_{1}\left(2^{-j_{1}} t_{1} \sqrt{L_{1}}\right) \Upsilon_{1}\left(2^{-j_{1}} t_{1} \sqrt{L_{1}}\right)}\left(y_{1}, z_{1}\right) \\
& \times K_{\Psi_{2}\left(2^{-j_{2}} t_{2} \sqrt{L_{2}}\right) \Upsilon_{2}\left(2^{-j_{2}} t_{2} \sqrt{L_{2}}\right)}\left(y_{2}, z_{2}\right) \\
& \times\left(\Phi_{1}\left(2^{-\left(0+j_{1}\right)} t_{1} \sqrt{L_{1}}\right) \otimes \Phi_{2}\left(2^{-\left(0+j_{2}\right)} t_{2} \sqrt{L_{1}}\right)\right) f\left(z_{1}, z_{2}\right) d \mu_{1}\left(z_{1}\right) d \mu_{2}\left(z_{2}\right) \\
& +\sum_{k_{1}=1}^{\infty} \iint_{X_{1} \times X_{2}} K_{\Phi_{1}\left(2^{-j_{1}} t_{1} \sqrt{L_{1}}\right) \Theta_{1}\left(2^{-\left(k_{1}+j_{1}\right)} t_{1} \sqrt{L_{1}}\right)}\left(y_{1}, z_{1}\right) \\
& \times K_{\Psi_{2}\left(2^{-j_{2}} t_{2} \sqrt{L_{2}}\right) \Upsilon_{2}\left(2^{-j_{2}} t_{2} \sqrt{L_{2}}\right)}\left(y_{2}, z_{2}\right) \\
& \times\left(\Phi_{1}\left(2^{-\left(k_{1}+j_{1}\right)} t_{1} \sqrt{L_{1}}\right) \otimes \Phi_{2}\left(2^{-\left(0+j_{2}\right)} t_{2} \sqrt{L_{1}}\right)\right) f\left(z_{1}, z_{2}\right) d \mu_{1}\left(z_{1}\right) d \mu_{2}\left(z_{2}\right) \\
& +\sum_{k_{2}=1}^{\infty} \iint_{X_{1} \times X_{2}} K_{\Psi_{1}\left(2^{-j_{1}} t_{1} \sqrt{L_{1}}\right) \Upsilon_{1}\left(2^{-j_{1}} t_{1} \sqrt{L_{1}}\right)}\left(y_{1}, z_{1}\right)
\end{aligned}
$$




$$
\begin{aligned}
& \quad \times K_{\Phi_{2}\left(2^{-j_{2}} t_{2} \sqrt{L_{2}}\right) \Theta_{2}\left(2^{-\left(k_{2}+j_{2}\right)} t_{2} \sqrt{L_{2}}\right)}\left(y_{2}, z_{2}\right) \\
& \quad \times\left(\Phi_{1}\left(2^{-\left(0+j_{1}\right)} t_{1} \sqrt{L_{1}}\right) \otimes \Phi_{2}\left(2^{-\left(k_{2}+j_{2}\right)} t_{2} \sqrt{L_{1}}\right)\right) f\left(z_{1}, z_{2}\right) d \mu_{1}\left(z_{1}\right) d \mu_{2}\left(z_{2}\right) \\
& +\sum_{k_{1}=1}^{\infty} \sum_{k_{2}=1}^{\infty} \iint_{X_{1} \times X_{2}} K_{\Phi_{1}\left(2^{-j_{1}} t_{1} \sqrt{L_{1}}\right) \Theta_{1}\left(2^{-\left(k_{1}+j_{1}\right)} t_{1} \sqrt{L_{1}}\right)}\left(y_{1}, z_{1}\right) \\
& \quad \times K_{\Phi_{2}\left(2^{-j_{2}} t_{2} \sqrt{L_{2}}\right) \Theta_{2}\left(2^{-\left(k_{2}+j_{2}\right)} t_{2} \sqrt{L_{2}}\right)}\left(y_{2}, z_{2}\right) \\
& \quad \times\left(\Phi_{1}\left(2^{-\left(k_{1}+j_{1}\right)} t_{1} \sqrt{L_{1}}\right) \otimes \Phi_{2}\left(2^{-\left(k_{2}+j_{2}\right)} t_{2} \sqrt{L_{2}}\right)\right) f\left(z_{1}, z_{2}\right) d \mu_{1}\left(z_{1}\right) d \mu_{2}\left(z_{2}\right) .
\end{aligned}
$$

For $i=1,2$, let $N_{i} \geq \lambda_{i}$ and $m_{i}$ be any integer such that $m_{i}-\lambda_{i}-n_{i} / r>0$. Since $\Theta_{i}$ vanishes near the origin, it follows from Lemma 2.2 that there exists a constant $C=C\left(\Phi_{i}, \Theta_{i}, m_{i}, N_{i}\right)$ such that for all $j_{i} \in \mathbb{Z}$, all $k_{i} \in\{1,2, \ldots\}$, and all $t_{i} \in[1,2]$,

$$
\begin{aligned}
& \left|K_{\Phi_{i}\left(2^{-j_{i}} t_{i} \sqrt{L_{i}}\right) \Theta_{i}\left(2^{-\left(k_{i}+j_{i}\right)} t_{i} \sqrt{L_{i}}\right)}\left(y_{i}, z_{i}\right)\right| \\
& \quad \leq C 2^{-k_{i} m_{i}} V\left(z_{i}, 2^{-j_{i}} t_{i}\right)^{-1}\left(1+2^{j_{i}} t_{i}^{-1} \rho_{i}\left(y_{i}, z_{i}\right)\right)^{-N_{i}} .
\end{aligned}
$$

Analogously, for $i=1,2$, we have

$$
\left|K_{\Psi_{i}\left(2^{-j_{i}} t_{i} \sqrt{L_{i}}\right) \Upsilon_{i}\left(2^{-j_{i}} t_{i} \sqrt{L_{i}}\right)}\left(y_{i}, z_{i}\right)\right| \leq C V\left(z_{i}, 2^{-j_{i}} t_{i}\right)^{-1}\left(1+2^{j_{i}} t_{i}^{-1} \rho_{i}\left(y_{i}, z_{i}\right)\right)^{-N_{i}} .
$$

Putting (3.8) and (3.9) into (3.7), we obtain

$$
\begin{aligned}
\left|\Phi_{1}\left(2^{-j_{1}} t_{1} \sqrt{L_{1}}\right) \otimes \Phi_{2}\left(2^{-j_{2}} t_{2} \sqrt{L_{2}}\right) f\left(y_{1}, y_{2}\right)\right| \\
\leq C \sum_{k_{1}=0}^{\infty} \sum_{k_{2}=0}^{\infty} 2^{-k_{1} m_{1}} 2^{-k_{2} m_{2}} \\
\quad \times \iint_{X_{1} \times X_{2}} \frac{\left|\Phi_{1}\left(2^{-\left(k_{1}+j_{1}\right)} t_{1} \sqrt{L_{1}}\right) \otimes \Phi_{2}\left(2^{-\left(k_{2}+j_{2}\right)} t_{2} \sqrt{L_{2}}\right) f\left(z_{1}, z_{2}\right)\right|}{\prod_{i=1}^{2} V\left(z_{i}, 2^{-j_{i}} t_{i}\right)\left(1+2^{j_{i}} t_{i}^{-1} \rho_{i}\left(y_{i}, z_{i}\right)\right)^{N_{i}}} d \mu_{1}\left(z_{1}\right) d \mu_{2}\left(z_{2}\right) \\
=C \sum_{k_{1}=j_{1}}^{\infty} \sum_{k_{2}=j_{2}}^{\infty} 2^{\left(j_{1}-k_{1}\right) m_{1}} 2^{\left(j_{2}-k_{2}\right) m_{2}} \\
\quad \times \iint_{X_{1} \times X_{2}} \frac{\left|\Phi_{1}\left(2^{-k_{1}} t_{1} \sqrt{L_{1}}\right) \otimes \Phi_{2}\left(2^{-k_{2}} t_{2} \sqrt{L_{2}}\right) f\left(z_{1}, z_{2}\right)\right|}{\prod_{i=1}^{2} V\left(z_{i}, 2^{-j_{i}} t_{i}\right)\left(1+2^{j_{i}} t_{i}^{-1} \rho_{i}\left(y_{i}, z_{i}\right)\right)^{N_{i}}} d \mu_{1}\left(z_{1}\right) d \mu_{2}\left(z_{2}\right) .
\end{aligned}
$$

To prove the desired inequality, we first consider the case $0<r \leq 1$. Dividing both sides of $(3.10)$ by $\left(1+2^{j_{1}} t_{1}^{-1} \rho_{1}\left(x_{1}, y_{1}\right)\right)^{\lambda_{1}}\left(1+2^{j_{2}} t_{2}^{-1} \rho_{2}\left(x_{2}, y_{2}\right)\right)^{\lambda_{2}}$, taking the supremum over $\left(y_{1}, y_{2}\right) \in X_{1} \times X_{2}$ in the left-hand side, and using the inequalities $V\left(z, 2^{-j_{i}} t_{i}\right) \geq V\left(z_{i}, 2^{-k_{i}} t_{i}\right)\left(\forall k_{i} \geq j_{i}\right)$ and $\left(1+2^{j_{i}} t_{i}^{-1} \rho_{i}\left(x_{i}, y_{i}\right)\right)\left(1+2^{j_{i}} t_{i}^{-1} \rho_{i}\left(y_{i}, z_{i}\right)\right) \geq$ $\left(1+2^{j_{i}} t_{i}^{-1} \rho_{i}\left(x_{i}, z_{i}\right)\right)\left(\forall t_{i} \in[1,2]\right)$ in the right-hand side, we get that, for all $t_{i} \in[1,2]$ and $x_{i} \in X_{i}$,

$$
\begin{aligned}
& {\left[\Phi_{1}\left(2^{-j_{1}} t_{1} \sqrt{L_{1}}\right) \otimes \Phi_{2}\left(2^{-j_{2}} t_{2} \sqrt{L_{2}}\right)\right]_{\lambda_{1}, \lambda_{2}}^{*} f\left(x_{1}, x_{2}\right)} \\
& \leq C \sum_{k_{1}=j_{1}}^{\infty} \sum_{k_{2}=j_{2}}^{\infty} 2^{\left(j_{1}-k_{1}\right) m_{1}} 2^{\left(j_{2}-k_{2}\right) m_{2}}
\end{aligned}
$$




$$
\times \iint_{X_{1} \times X_{2}} \frac{\left|\Phi_{1}\left(2^{-k_{1}} t_{1} \sqrt{L_{1}}\right) \otimes \Phi_{2}\left(2^{-k_{2}} t_{2} \sqrt{L_{2}}\right) f\left(z_{1}, z_{2}\right)\right|}{\prod_{i=1}^{2} V\left(z_{i}, 2^{-k_{i}} t_{i}\right)\left(1+2^{j_{i}} t_{i}^{-1} \rho_{i}\left(x_{i}, z_{i}\right)\right)^{\lambda_{i}}} d \mu_{1}\left(z_{1}\right) d \mu_{2}\left(z_{2}\right) .
$$

To proceed further, we note that

$$
\begin{aligned}
& \left|\Phi_{1}\left(2^{-k_{1}} t_{1} \sqrt{L_{1}}\right) \otimes \Phi_{2}\left(2^{-k_{2}} t_{2} \sqrt{L_{2}}\right) f\left(z_{1}, z_{2}\right)\right| \\
& \leq \mid \\
& \quad\left|\Phi_{1}\left(2^{-k_{1}} t_{1} \sqrt{L_{1}}\right) \otimes \Phi_{2}\left(2^{-k_{2}} t_{2} \sqrt{L_{2}}\right) f\left(z_{1}, z_{2}\right)\right|^{r} \\
& \quad \times\left\{\left[\Phi_{1}\left(2^{-k_{1}} t_{1} \sqrt{L_{1}}\right) \otimes \Phi_{2}\left(2^{-k_{2}} t_{2} \sqrt{L_{2}}\right)\right]_{\lambda_{1}, \lambda_{2}}^{*} f\left(x_{1}, x_{2}\right)\right\}^{1-r} \\
& \quad \times\left(1+2^{k_{1}} t_{1}^{-1} \rho_{1}\left(x_{1}, z_{1}\right)\right)^{\lambda_{1}(1-r)}\left(1+2^{k_{2}} t_{2}^{-1} \rho_{2}\left(x_{2}, z_{2}\right)\right)^{\lambda_{2}(1-r)} .
\end{aligned}
$$

From (3.11), (3.12), and the inequality

$$
\left(1+2^{k_{i}} t_{i}^{-1} \rho_{i}\left(x_{i}, z_{i}\right)\right)^{\lambda_{i}} \leq 2^{\left(k_{i}-j_{i}\right) \lambda_{i}}\left(1+2^{j_{i}} t_{i}^{-1} \rho_{i}\left(x_{i}, z_{i}\right)\right)^{\lambda_{i}} \quad\left(\forall k_{i} \geq j_{i}, \forall t_{i} \in[1,2]\right),
$$

it follows that

$$
\begin{aligned}
& {\left[\Phi_{1}\left(2^{-j_{1}} t_{1} \sqrt{L_{1}}\right) \otimes \Phi_{2}\left(2^{-j_{2}} t_{2} \sqrt{L_{2}}\right)\right]_{\lambda_{1}, \lambda_{2}}^{*} f\left(x_{1}, x_{2}\right)} \\
& \leq C \sum_{k_{1}=j_{1}}^{\infty} \sum_{k_{2}=j_{2}}^{\infty} 2^{\left(j_{1}-k_{1}\right)\left(m_{1}-\lambda_{1}\right)} 2^{\left(j_{2}-k_{2}\right)\left(m_{2}-\lambda_{2}\right)} \\
& \quad \times \iint_{X_{1} \times X_{2}} \frac{\left|\Phi_{1}\left(2^{-k_{1}} t_{1} \sqrt{L_{1}}\right) \otimes \Phi_{2}\left(2^{-k_{2}} t_{2} \sqrt{L_{2}}\right) f\left(z_{1}, z_{2}\right)\right|^{r}}{\prod_{i=1}^{2} V\left(z_{i}, 2^{-k_{i}} t_{i}\right)\left(1+2^{k_{i}} t_{i}^{-1} \rho_{i}\left(x_{i}, z_{i}\right)\right)^{\lambda_{i} r}} d \mu_{1}\left(z_{1}\right) d \mu_{2}\left(z_{2}\right) \\
& \quad \times\left\{\left[\Phi_{1}\left(2^{-k_{1}} t_{1} \sqrt{L_{1}}\right) \otimes \Phi_{2}\left(2^{-k_{2}} t_{2} \sqrt{L_{2}}\right)\right]_{\lambda_{1}, \lambda_{2}}^{*} f\left(x_{1}, x_{2}\right)\right\}^{1-r} .
\end{aligned}
$$

We claim that for any $f \in L^{2}\left(X_{1} \times X_{2}\right), \lambda_{i}>D_{i} / 2, x_{i} \in X_{i}, t_{i} \in[1,2]$, and $j_{i} \in \mathbb{Z}$,

$$
\left[\Phi_{1}\left(2^{-j_{1}} t_{1} \sqrt{L_{1}}\right) \otimes \Phi_{2}\left(2^{-j_{2}} t_{2} \sqrt{L_{2}}\right)\right]_{\lambda_{1}, \lambda_{2}}^{*} f\left(x_{1}, x_{2}\right)<\infty,
$$

and there exists $N_{0}>0$ such that

$$
\left[\Phi_{1}\left(2^{-j_{1}} t_{1} \sqrt{L_{1}}\right) \otimes \Phi_{2}\left(2^{-j_{2}} t_{2} \sqrt{L_{2}}\right)\right]_{\lambda_{1}, \lambda_{2}}^{*} f\left(x_{1}, x_{2}\right)=O\left(2^{j_{1} N_{0}} 2^{j_{2} N_{0}}\right)
$$

as $j_{1}, j_{2} \rightarrow+\infty$. Indeed, for $i=1,2$, by Lemma 2.1 we have

$$
\left|K_{\Phi_{i}\left(2^{-j_{i}} t_{i} \sqrt{L_{i}}\right)}\left(y_{i}, z_{i}\right)\right| \leq C V\left(y_{i}, 2^{-j_{i}} t_{i}\right)^{-1}\left(1+2^{j_{i}} t_{i}^{-1} \rho\left(y_{i}, z_{i}\right)\right)^{-\left(n_{i}+1\right) / 2} .
$$

Hence, by the Cauchy-Schwartz inequality and (2.1), we have

$$
\begin{aligned}
& \left|\Phi_{1}\left(2^{-j_{1}} t_{1} \sqrt{L_{1}}\right) \otimes \Phi_{2}\left(2^{-j_{2}} t_{2} \sqrt{L_{2}}\right) f\left(y_{1}, y_{2}\right)\right| \\
& \quad \leq C \iint_{X_{1} \times X_{2}}\left|K_{\Phi_{1}\left(2^{-j_{1}} t_{1} \sqrt{L_{1}}\right)}\left(y_{1}, z_{1}\right)\right|\left|K_{\Phi_{2}\left(2^{-j_{2}} t_{2} \sqrt{L_{2}}\right)}\left(y_{2}, z_{2}\right)\right|\left|f\left(z_{1}, z_{2}\right)\right| d \mu_{1}\left(z_{1}\right) d \mu_{2}\left(z_{2}\right) \\
& \quad \leq C\|f\|_{L^{2}\left(X_{1} \times X_{2}\right)} V\left(y_{1}, 2^{-j_{1}} t_{1}\right)^{-1 / 2} V\left(y_{2}, 2^{-j_{2}} t_{1}\right)^{-1 / 2} .
\end{aligned}
$$


This along with (1.3) yields that for $\lambda_{i} \geq D_{i} / 2$,

$$
\begin{aligned}
& {\left[\Phi_{1}\left(2^{-j_{1}} t_{1} \sqrt{L_{1}}\right) \otimes \Phi_{2}\left(2^{-j_{2}} t_{2} \sqrt{L_{2}}\right)\right]_{\lambda_{1}, \lambda_{2}}^{*} f\left(x_{1}, x_{2}\right)} \\
& \quad \leq C \sup _{\left(y_{1}, y_{2}\right) \in X_{1} \times X_{2}} \frac{\|f\|_{L^{2}\left(X_{1} \times X_{2}\right)}}{\prod_{i=1}^{2} V\left(y_{i}, 2^{-j_{i}} t_{i}\right)^{-1 / 2}\left(1+2^{j_{i}} t_{i}^{-1} \rho_{i}\left(x_{i}, y_{i}\right)\right)^{\lambda_{i}}} \\
& \quad \leq C\|f\|_{L^{2}\left(X_{1} \times X_{2}\right)} V\left(x_{1}, 2^{-j_{1}} t_{1}\right)^{-1 / 2} V\left(x_{2}, 2^{-j_{2}} t_{2}\right)^{-1 / 2} .
\end{aligned}
$$

Hence (3.14) is true. Moreover, if $j_{1}, j_{2} \geq 1$, by (1.2) we have

$$
\begin{aligned}
& {\left[\Phi_{1}\left(2^{-j_{1}} t_{1} \sqrt{L_{1}}\right) \otimes \Phi_{2}\left(2^{-j_{2}} t_{2} \sqrt{L_{2}}\right)\right]_{\lambda_{1}, \lambda_{2}}^{*} f\left(x_{1}, x_{2}\right)} \\
& \quad \leq C\|f\|_{L^{2}\left(X_{1} \times X_{2}\right)} V\left(x_{1}, 2^{-j_{1}} t_{1}\right)^{-1 / 2} V\left(x_{2}, 2^{-j_{2}} t_{2}\right)^{-1 / 2} \\
& \quad \leq C 2^{j_{1} n_{1} / 2} 2^{j_{2} n_{2} / 2}\|f\|_{L^{2}\left(X_{1} \times X_{2}\right)} V\left(x_{1}, 1\right)^{-1 / 2} V\left(x_{2}, 1\right)^{-1 / 2},
\end{aligned}
$$

which verifies (3.15) with $N_{0}=\max \left\{n_{1} / 2, n_{2} / 2\right\}$.

Since $m_{1}, m_{2}$ in (3.13) can be chosen to be arbitrarily large, it follows from (3.13),

(3.14), (3.15) and Lemma 2.7 that for any $\sigma>0$,

$$
\begin{aligned}
& \left\{\left[\Phi_{1}\left(2^{-j_{1}} t_{1} \sqrt{L_{1}}\right) \otimes \Phi_{2}\left(2^{-j_{2}} t_{2} \sqrt{L_{2}}\right)\right]_{\lambda_{1}, \lambda_{2}}^{*} f\left(x_{1}, x_{2}\right)\right\}^{r} \\
& \leq C \sum_{k_{1}=j_{1}}^{\infty} \sum_{k_{2}=j_{2}}^{\infty} 2^{\left(j_{1}-k_{1}\right) \sigma} 2^{\left(j_{2}-k_{2}\right) \sigma} \iint_{X_{1} \times X_{2}} \\
& \quad \times \frac{\left|\Phi_{1}\left(2^{-k_{1}} t_{1} \sqrt{L_{1}}\right) \otimes \Phi_{2}\left(2^{-k_{2}} t_{2} \sqrt{L_{2}}\right) f\left(z_{1}, z_{2}\right)\right|^{r} d \mu_{1}\left(z_{1}\right) d \mu_{2}\left(z_{2}\right)}{V\left(z_{1}, 2^{-k_{1}} t_{1}\right)\left(1+2^{k_{1}} t_{1}^{-1} \rho_{1}\left(x_{1}, z_{1}\right)\right)^{\lambda_{1} r} V\left(z_{2}, 2^{-k_{2}} t_{2}\right)\left(1+2^{k_{2}} t_{2}^{-1} \rho_{2}\left(x_{2}, z_{2}\right)\right)^{\lambda_{2} r}} .
\end{aligned}
$$

This proves $(3.5)$ for $0<r \leq 1$.

Next we show (3.5) for $r>1$. Indeed, from (3.10) with $m_{i} \geq \sigma+\lambda_{i} r+\varepsilon$ and $N_{i} \geq \lambda_{i}+\left(D_{i}+n_{i}+1\right) / r^{\prime}$, where $\varepsilon$ is any fixed positive number and $r^{\prime}$ is a number such that $1 / r+1 / r^{\prime}=1$, it follows that

$$
\begin{aligned}
&\left|\Phi_{1}\left(2^{-j_{1}} t_{1} \sqrt{L_{1}}\right) \otimes \Phi_{2}\left(2^{-j_{2}} t_{2} \sqrt{L_{2}}\right) f\left(y_{1}, y_{2}\right)\right| \\
& \leq C \sum_{k_{1}=j_{1}}^{\infty} \sum_{k_{2}=j_{2}}^{\infty} 2^{\left(j_{1}-k_{1}\right)\left(\sigma+\lambda_{1} r+\varepsilon\right)} 2^{\left(j_{2}-k_{2}\right)\left(\sigma+\lambda_{2} r+\varepsilon\right)} \\
& \quad \times \iint_{X_{1} \times X_{2}} \frac{\left|\Phi_{1}\left(2^{-k_{1}} t_{1} \sqrt{L_{1}}\right) \otimes \Phi_{2}\left(2^{-k_{2}} t_{2} \sqrt{L_{2}}\right) f\left(z_{1}, z_{2}\right)\right|}{\prod_{i=1}^{2} V\left(z_{i}, 2^{-j_{i}} t_{i}\right)\left(1+2^{j_{i}} t_{i}^{-1} \rho_{i}\left(y_{i}, z_{i}\right)\right)^{\lambda_{i}+\left(D_{i}+n_{i}+1\right) / r^{\prime}}} d \mu_{1}\left(z_{1}\right) d \mu_{2}\left(z_{2}\right) \\
& \leq C \sum_{k_{1}=j_{1}}^{\infty} \sum_{k_{2}=j_{2}}^{\infty} 2^{\left(j_{1}-k_{1}\right)\left(\sigma+\lambda_{1} r+\varepsilon\right)} 2^{\left(j_{2}-k_{2}\right)\left(\sigma+\lambda_{2} r+\varepsilon\right)} \\
& \quad \times\left(\iint_{X_{1} \times X_{2}} \frac{\left|\Phi_{1}\left(2^{-k_{1}} t_{1} \sqrt{L_{1}}\right) \otimes \Phi_{2}\left(2^{-k_{2}} t_{2} \sqrt{L_{2}}\right) f\left(z_{1}, z_{2}\right)\right|^{r}}{\prod_{i=1}^{2} V\left(z_{i}, 2^{-j_{i}} t_{i}\right)\left(1+2^{j_{i}} t_{i}^{-1} \rho_{i}\left(y_{i}, z_{i}\right)\right)^{\lambda_{i} r}} d \mu_{1}\left(z_{1}\right) d \mu_{2}\left(z_{2}\right)\right)^{1 / r} \\
& \leq C\left(\sum_{k_{1}=j_{1}}^{\infty} \sum_{k_{2}=j_{2}}^{\infty} 2^{\left(j_{1}-k_{1}\right)\left(\sigma_{1}+\lambda_{1} r\right)} 2^{\left(j_{2}-k_{2}\right)\left(\sigma_{2}+\lambda_{2} r\right)}\right.
\end{aligned}
$$




$$
\left.\times \iint_{X_{1} \times X_{2}} \frac{\left|\Phi_{1}\left(2^{-k_{1}} t_{1} \sqrt{L_{1}}\right) \otimes \Phi_{2}\left(2^{-k_{2}} t_{2} \sqrt{L_{2}}\right) f\left(z_{1}, z_{2}\right)\right|^{r}}{\prod_{i=1}^{2} V\left(z_{i}, 2^{-j_{i}} t_{i}\right)\left(1+2^{j_{i}} t_{i}^{-1} \rho_{i}\left(y_{i}, z_{i}\right)\right)^{\lambda_{i} r}} d \mu_{1}\left(z_{1}\right) d \mu_{2}\left(z_{2}\right)\right)^{1 / r},
$$

where we applied Hölder's inequality for the integrals and the sums, and used (1.3) and (2.1). Raising both sides to the power $r$, dividing both sides by $(1+$ $\left.2^{j_{1}} t_{1}^{-1} \rho_{1}\left(x_{1}, y_{1}\right)\right)^{\lambda_{1} r}\left(1+2^{j_{2}} t_{2}^{-1} \rho_{2}\left(x_{2}, y_{2}\right)\right)^{\lambda_{2} r}$, in the left-hand side taking the supremum over $\left(y_{1}, y_{2}\right) \in X_{1} \times X_{2}$, and in the right-hand side using the inequalities

$$
\begin{aligned}
(1 & \left.+2^{j_{i}} t_{i}^{-1} \rho_{i}\left(x_{i}, y_{i}\right)\right)^{\lambda_{i} r}\left(1+2^{j_{i}} t_{i}^{-1} \rho_{i}\left(y_{i}, z_{i}\right)\right)^{\lambda_{i} r} \\
& \geq\left(1+2^{j_{i}} t_{i}^{-1} \rho_{i}\left(x_{i}, z_{i}\right)\right)^{\lambda_{i} r} \\
& \geq 2^{\left(j_{i}-k_{i}\right) \lambda_{i} r}\left(1+2^{k_{i}} t_{i}^{-1} \rho_{i}\left(x_{i}, z_{i}\right)\right)^{\lambda_{i} r} \quad\left(\forall k_{i} \geq j_{i}\right)
\end{aligned}
$$

and $V\left(z_{i}, 2^{-j_{i}} t_{i}\right) \geq V\left(z_{i}, 2^{-k_{i}} t_{i}\right)\left(\forall k_{i} \geq j_{i}\right)$, we obtain (3.5) for $r>1$.

Lemma 3.5. Let $\Phi_{1}, \Phi_{2} \in \mathcal{A}(\mathbb{R})$ be even functions. Let $p \in(0, \infty)$ and $\lambda_{i}>$ $\left(n_{i}+D_{i}\right) q_{w} / \min \{p, 2\}, i=1,2$. Then there exists a constant $C$ such that for all $f \in$ $L^{2}\left(X_{1} \times X_{2}\right)$,

$$
\begin{gathered}
\left\|\left(\int_{0}^{\infty} \int_{0}^{\infty}\left|\left[\Phi_{1}\left(t_{1} \sqrt{L_{1}}\right) \otimes \Phi_{2}\left(t_{2} \sqrt{L_{2}}\right)\right]_{\lambda_{1}, \lambda_{2}}^{*} f\right|^{2} \frac{d t_{1}}{t_{1}} \frac{d t_{2}}{t_{2}}\right)^{1 / 2}\right\|_{L_{w}^{p}\left(X_{1} \times X_{2}\right)} \\
\leq C\left\|\left(\int_{0}^{\infty} \int_{0}^{\infty}\left|\Phi_{1}\left(t_{1} \sqrt{L_{1}}\right) \otimes \Phi_{2}\left(t_{2} \sqrt{L_{2}}\right) f\right|^{2} \frac{d t_{1}}{t_{1}} \frac{d t_{2}}{t_{2}}\right)^{1 / 2}\right\|_{L_{w}^{p}\left(X_{1} \times X_{2}\right)} .
\end{gathered}
$$

Proof. Since $\lambda_{i}>\left(n_{i}+D_{i}\right) q_{w} / \min \{p, 2\}$, there exists a number $r$ such that $0<r<\min \{p, 2\} / q_{w}$ and $\lambda_{i} r>n_{i}+D_{i}$. From Lemma 3.4 we see that for any $\sigma>0$ there exists a constant $C$ such that for all $f \in L^{2}\left(X_{1} \times X_{2}\right), j_{i} \in \mathbb{Z}, x_{i} \in X_{i}$ and $t_{i} \in[1,2]$,

$$
\begin{aligned}
& \left\{\left[\Phi_{1}\left(2^{-j_{1}} t_{1} \sqrt{L_{1}}\right) \otimes \Phi_{2}\left(2^{-j_{2}} t_{2} \sqrt{L_{2}}\right)\right]_{\lambda_{1}, \lambda_{2}}^{*} f\left(x_{1}, x_{2}\right)\right\}^{r} \\
& \leq C \sum_{k_{1}=j_{1}}^{\infty} \sum_{k_{2}=j_{2}}^{\infty} 2^{\left(j_{1}-k_{1}\right) \sigma_{2}\left(j_{2}-k_{2}\right) \sigma} \\
& \times \iint_{X_{1} \times X_{2}} \frac{\left|\Phi_{1}\left(2^{-k_{1}} t_{1} \sqrt{L_{1}}\right) \otimes \Phi_{2}\left(2^{-k_{2}} t_{2} \sqrt{L_{2}}\right) f\left(z_{1}, z_{2}\right)\right|^{r} d \mu_{1}\left(z_{1}\right) d \mu_{2}\left(z_{2}\right)}{V\left(z_{1}, 2^{-k_{1}} t_{1}\right)\left(1+2^{k_{1}} t_{1}^{-1} \rho_{1}\left(x_{1}, z_{1}\right)\right)^{\lambda_{1} r} V\left(z_{2}, 2^{-k_{2}} t_{2}\right)\left(1+2^{k_{2}} t_{2}^{-1} \rho_{2}\left(x_{2}, z_{2}\right)\right)^{\lambda_{2} r}} .
\end{aligned}
$$

Taking the norm $\left(\left.\int_{1}^{2} \int_{1}^{2}|\cdot|\right|^{2 / r}\left(d t_{1} / t_{1}\right)\left(d t_{2} / t_{2}\right)\right)^{r / 2}$ on both sides, applying Minkowski's inequality, and then using (2.7), we get

$$
\begin{aligned}
& \left(\int_{1}^{2} \int_{1}^{2}\left|\left[\Phi_{1}\left(2^{-j_{1}} t_{1} \sqrt{L_{1}}\right) \otimes \Phi_{2}\left(2^{-j_{2}} t_{2} \sqrt{L_{2}}\right)\right]_{\lambda_{1}, \lambda_{2}}^{*} f\left(x_{1}, x_{2}\right)\right|^{2} \frac{d t_{1}}{t_{1}} \frac{d t_{2}}{t_{2}}\right)^{r / 2} \\
& \leq C \sum_{k_{1}=j_{1}}^{\infty} \sum_{k_{2}=j_{2}}^{\infty} 2^{\left(j_{1}-k_{1}\right) \sigma} 2^{\left(j_{2}-k_{2}\right) \sigma}
\end{aligned}
$$


$\times \iint_{X_{1} \times X_{2}} \frac{\left(\int_{1}^{2} \int_{1}^{2}\left|\Phi_{1}\left(2^{-k_{1}} t_{1} \sqrt{L_{1}}\right) \otimes \Phi_{2}\left(2^{-k_{2}} t_{2} \sqrt{L_{2}}\right) f\left(z_{1}, z_{2}\right)\right|^{2} \frac{d t_{1}}{t_{1}} \frac{d t_{2}}{t_{2}}\right)^{r / 2} d \mu_{1}\left(z_{1}\right) d \mu_{2}\left(z_{2}\right)}{V\left(z_{1}, 2^{-k_{1}} t_{1}\right)\left(1+2^{k_{1}} t_{1}^{-1} \rho_{1}\left(x_{1}, z_{1}\right)\right)^{\lambda_{1} r} V\left(z_{2}, 2^{-k_{2}} t_{2}\right)\left(1+2^{k_{2}} t_{2}^{-1} \rho_{2}\left(x_{2}, z_{2}\right)\right)^{\lambda_{2} r}}$ $\leq C \sum_{k_{1}=j_{1}}^{\infty} \sum_{k_{2}=j_{2}}^{\infty} 2^{\left(j_{1}-k_{1}\right) \sigma} 2^{\left(j_{2}-k_{2}\right) \sigma}$

$\times \mathcal{M}_{s}\left[\left(\int_{1}^{2} \int_{1}^{2}\left|\Phi_{1}\left(2^{-k_{1}} t_{1} \sqrt{L_{1}}\right) \otimes \Phi_{2}\left(2^{-k_{2}} t_{2} \sqrt{L_{2}}\right) f\right|^{2} \frac{d t_{1}}{t_{1}} \frac{d t_{2}}{t_{2}}\right)^{r / 2}\right]\left(x_{1}, x_{2}\right)$

$\leq C \sum_{k_{1}=-\infty}^{\infty} \sum_{k_{2}=-\infty}^{\infty} 2^{-\left|k_{1}-j_{1}\right| \sigma_{2}} 2^{-\left|k_{2}-j_{2}\right| \sigma}$

$\times \mathcal{M}_{s}\left[\left(\int_{1}^{2} \int_{1}^{2}\left|\Phi_{1}\left(2^{-k_{1}} t_{1} \sqrt{L_{1}}\right) \otimes \Phi_{2}\left(2^{-k_{2}} t_{2} \sqrt{L_{2}}\right) f\right|^{2} \frac{d t_{1}}{t_{1}} \frac{d t_{2}}{t_{2}}\right)^{r / 2}\right]\left(x_{1}, x_{2}\right)$.

It then follows from Lemma 2.6 and Lemma 2.8 that

$$
\begin{aligned}
& \left\|\left(\int_{0}^{\infty} \int_{0}^{\infty}\left|\left[\Phi_{1}\left(t_{1} \sqrt{L_{1}}\right) \otimes \Phi_{2}\left(t_{2} \sqrt{L_{2}}\right)\right]_{\lambda_{1}, \lambda_{2}}^{*} f\right|^{2} \frac{d t_{1}}{t_{1}} \frac{d t_{2}}{t_{2}}\right)^{1 / 2}\right\|_{L_{w}^{p}\left(X_{1} \times X_{2}\right)} \\
& =\left\|\left\{\left(\int_{1}^{2} \int_{1}^{2}\left|\left[\Phi_{1}\left(2^{-j_{1}} t_{1} \sqrt{L_{1}}\right) \otimes \Phi_{2}\left(2^{-j_{2}} t_{2} \sqrt{L_{2}}\right)\right]_{\lambda_{1}, \lambda_{2}}^{*} f\right|^{2} \frac{d t_{1}}{t_{1}} \frac{d t_{2}}{t_{2}}\right)^{r / 2}\right\}_{j_{1}, j_{2} \in \mathbb{Z}}\right\|_{L_{w}^{p / r}\left(\ell^{2 / r}\right)}^{1 / r} \\
& \left.\leq C\left\|\left\{\mathcal{M}_{s}\left[\left(\int_{1}^{2} \int_{1}^{2}\left|\Phi_{1}\left(2^{-j_{1}} t_{1} \sqrt{L_{1}}\right) \otimes \Phi_{2}\left(2^{-j_{2}} t_{2} \sqrt{L_{2}}\right) f\right|^{2} \frac{d t_{1}}{t_{1}} \frac{d t_{2}}{t_{2}}\right)^{r / 2}\right]\right\}_{j_{1}, j_{2} \in \mathbb{Z}}^{r / r}\right\|_{L_{w}^{p / r}\left(\ell^{2 / r}\right)}^{1 / r} \|\left\{\int_{1}^{2} \int_{1}^{2}\left|\Phi_{1}\left(2^{-j_{1}} t_{1} \sqrt{L_{1}}\right) \otimes \Phi_{2}\left(2^{-j_{2}} t_{2} \sqrt{L_{2}}\right) f\right|^{2} \frac{d t_{1}}{t_{1}} \frac{d t_{2}}{t_{2}}\right)^{r / 2}\right\} \\
& \leq C\left\|\left(\int_{0}^{\infty} \int_{0}^{\infty}\left|\Phi_{1}\left(t_{1} \sqrt{L_{1}}\right) \otimes \Phi_{2}\left(t_{2} \sqrt{L_{2}}\right) f\right|^{2} \frac{d t_{1}}{t_{1}} \frac{d t_{2}}{t_{2}}\right)^{1 / 2}\right\|\left\|_{L_{w}^{p / r}\left(\ell^{2 / r}\right)}\right\|_{L_{w}^{p}\left(X_{1} \times X_{2}\right)},
\end{aligned}
$$

where we used the fact that $p / r>q_{w}\left(\right.$ which implies $\left.w \in A_{p / r}\left(X_{1} \times X_{2}\right)\right)$ and $2 / r>1$.

Lemma 3.6. Let $\Phi_{1}, \Phi_{2} \in \mathcal{A}(\mathbb{R})$ be even functions. Let $p \in(0, \infty)$ and $\lambda_{i}>0$, $i=1,2$. Let $w$ be an arbitrary weight (i.e., nonnegative locally integrable function) on $X_{1} \times X_{2}$. Then there exists a constant $C$ such that for all $f \in L^{2}\left(X_{1} \times X_{2}\right)$,

$$
\begin{aligned}
& \left\|\left(\int_{0}^{\infty} \int_{0}^{\infty}\left|\left[\Phi_{1}\left(t_{1} \sqrt{L_{1}}\right) \otimes \Phi_{2}\left(t_{2} \sqrt{L_{2}}\right)\right]_{\lambda_{1}+D_{1} / 2, \lambda_{2}+D_{2} / 2}^{*} f\right|^{2} \frac{d t_{1}}{t_{1}} \frac{d t_{2}}{t_{2}}\right)^{1 / 2}\right\| \|_{L_{w}^{p}\left(X_{1} \times X_{2}\right)} \\
& \quad \leq C\left\|g_{\Phi_{1}, \Phi_{2}, L_{1}, L_{2},\left(2 / n_{1}\right) \lambda_{1},\left(2 / n_{2}\right) \lambda_{2}}^{*}(f)\right\|_{L_{w}^{p}\left(X_{1} \times X_{2}\right)} .
\end{aligned}
$$

Proof. Let $\sigma>0$. By Lemma 3.4 with $r=2$, we see that there exists a constant $C$ such that for all $f \in L^{2}\left(X_{1} \times X_{2}\right), j_{i} \in \mathbb{Z}$ and $t_{i} \in[1,2]$, 


$$
\begin{aligned}
& \left\{\left[\Phi_{1}\left(2^{-j_{1}} t_{1} \sqrt{L_{1}}\right) \otimes \Phi_{2}\left(2^{-j_{2}} t_{2} \sqrt{L_{2}}\right)\right]_{\lambda_{1}+D_{1} / 2, \lambda_{2}+D_{2} / 2}^{*} f\left(x_{1}, x_{2}\right)\right\}^{2} \\
& \leq C \sum_{k_{1}=j_{1}}^{\infty} \sum_{k_{2}=j_{2}}^{\infty} 2^{\left(j_{1}-k_{1}\right) \sigma} 2^{\left(j_{2}-k_{2}\right) \sigma} \\
& \quad \times \iint_{X_{1} \times X_{2}} \frac{\left|\Phi_{1}\left(2^{-k_{1}} t_{1} \sqrt{L_{1}}\right) \otimes \Phi_{2}\left(2^{-k_{2}} t_{2} \sqrt{L_{2}}\right) f\left(z_{1}, z_{2}\right)\right|^{2}}{\prod_{i=1}^{2} V\left(z_{i}, 2^{-k_{i}} t_{i}\right)\left(1+2^{k_{i}} t_{i}^{-1} \rho_{i}\left(x_{i}, z_{i}\right)\right)^{2 \lambda_{i}+D_{i}}} d \mu_{1}\left(z_{1}\right) d \mu_{2}\left(z_{2}\right) \\
& \leq C \sum_{k_{1}=-\infty}^{\infty} \sum_{k_{2}=-\infty}^{\infty} 2^{-\left|k_{1}-j_{1}\right| \sigma} 2^{-\left|k_{2}-j_{2}\right| \sigma} \\
& \quad \times \iint_{X_{1} \times X_{2}} \frac{\left|\Phi_{1}\left(2^{-k_{1}} t_{1} \sqrt{L_{1}}\right) \otimes \Phi_{2}\left(2^{-k_{2}} t_{2} \sqrt{L_{2}}\right) f\left(z_{1}, z_{2}\right)\right|^{2}}{\prod_{i=1}^{2}\left(1+2^{k_{i}} t_{i}^{-1} \rho\left(x_{i}, z_{i}\right)\right)^{2 \lambda_{i}}} \\
& \times \frac{d \mu_{1}\left(z_{1}\right) d \mu_{2}\left(z_{2}\right)}{V\left(x_{1}, 2^{-k_{1}} t_{1}\right) V\left(x_{2}, 2^{\left.-k_{2} t_{2}\right)}\right.},
\end{aligned}
$$

where for the last line we used (1.3). Taking the norm $\int_{1}^{2} \int_{1}^{2}|\cdot|\left(d t_{1} / t_{1}\right)\left(d t_{2} / t_{2}\right)$ on both sides of (3.16) gives

$$
\begin{gathered}
\int_{1}^{2} \int_{1}^{2}\left\{\left[\Phi_{1}\left(2^{-2 j_{1}} t_{1} \sqrt{L_{1}}\right) \otimes \Phi_{2}\left(2^{-j_{2}} t_{2} \sqrt{L_{2}}\right)\right]_{\lambda_{1}+D_{1} / 2, \lambda_{2}+D_{2} / 2}^{*} f\left(x_{1}, x_{2}\right)\right\}^{2} \frac{d t_{1}}{t_{1}} \frac{d t_{2}}{t_{2}} \\
\leq C \sum_{k_{1}=-\infty}^{\infty} \sum_{k_{2}=-\infty}^{\infty} 2^{-\left|k_{1}-j_{1}\right| \sigma} 2^{-\left|k_{2}-j_{2}\right| \sigma} \\
\quad \times \int_{1}^{2} \int_{1}^{2} \int_{X_{1}} \int_{X_{2}} \frac{\left|\Phi_{1}\left(2^{-k_{1}} t_{1} \sqrt{L_{1}}\right) \otimes \Phi_{2}\left(2^{-k_{2}} t_{2} \sqrt{L_{2}}\right) f\left(z_{1}, z_{2}\right)\right|^{2}}{\left(1+2^{k_{1}} t_{1}^{-1} \rho\left(x_{1}, z_{1}\right)\right)^{2 \lambda_{1}}\left(1+2^{k_{2}} t_{2}^{-1} \rho_{2}\left(x_{2}, z_{2}\right)\right)^{2 \lambda_{2}}} \\
\times \frac{d \mu_{1}\left(z_{1}\right) d t_{1} d \mu_{2}\left(z_{2}\right) d t_{2}}{V\left(x_{1}, 2^{-k_{1}} t_{1}\right) t_{1} V\left(x_{2}, 2^{-k_{2}} t_{2}\right) t_{2}} .
\end{gathered}
$$

Applying Lemma 2.6 in $L_{w}^{p / 2}\left(\ell^{1}\right)$ we obtain

$$
\begin{aligned}
& \left\|\left(\int_{0}^{\infty} \int_{0}^{\infty}\left|\left[\Phi_{1}\left(t_{1} \sqrt{L_{1}}\right) \otimes \Phi_{2}\left(t_{2} \sqrt{L_{2}}\right)\right]_{\lambda_{1}+D_{1} / 2, \lambda_{2}+D_{2} / 2}^{*} f\right|^{2} \frac{d t_{1}}{t_{1}} \frac{d t_{2}}{t_{2}}\right)^{1 / 2}\right\|_{L_{w}^{p}\left(X_{1} \times X_{2}\right)} \\
& =\|\left\{\int_{1}^{2} \int_{1}^{2}\left\{\left[\Phi_{1}\left(2^{-j_{1}} t_{1} \sqrt{L_{1}}\right) \otimes \Phi_{2}\left(2^{-j_{2}} t_{2} \sqrt{L_{2}}\right)\right]_{\lambda_{1}+D_{1} / 2, \lambda_{2}+D_{2} / 2}^{*} f\left(x_{1}, x_{2}\right)\right\}^{2}\right. \\
& \left.\times \frac{d t_{1}}{t_{1}} \frac{d t_{2}}{t_{2}}\right\}_{j_{1}, j_{2} \in \mathbb{Z}} \|_{L_{w}^{p / 2}\left(\ell^{1}\right)}^{1 / 2} \\
& \leq C \|\left\{\int_{1}^{2} \int_{1}^{2} \int_{X} \int_{X} \frac{\left|\Phi_{1}\left(2^{-k_{1}} t_{1} \sqrt{L_{1}}\right) \otimes \Phi_{2}\left(2^{-k_{2}} t_{2} \sqrt{L_{2}}\right) f\left(z_{1}, z_{2}\right)\right|^{2}}{\left(1+2^{k_{1}} t_{1}^{-1} \rho\left(x_{1}, z_{1}\right)\right)^{2 \lambda_{1}}\left(1+2^{k_{2}} t_{2}^{-1} \rho_{2}\left(x_{2}, z_{2}\right)\right)^{2 \lambda_{2}}}\right. \\
& \left.\times \frac{d \mu_{1}\left(z_{1}\right) d t_{1}}{V\left(x_{1}, 2^{-k_{1}} t_{1}\right) t_{1}} \frac{d \mu_{2}\left(z_{2}\right) d t_{2}}{V\left(x_{2}, 2^{-k_{2}} t_{2}\right) t_{2}}\right\}_{k_{1}, k_{2} \in \mathbb{Z}} \|_{L_{w}^{p / 2}\left(\ell^{1}\right)}^{1 / 2} \\
& =C\left\|g_{\Phi_{1}, \Phi_{2}, L_{1}, L_{2},\left(2 / n_{1}\right) \lambda_{1},\left(2 / n_{2}\right) \lambda_{2}}^{*}(f)\right\|_{L_{w}^{p}\left(X_{1} \times X_{2}\right)},
\end{aligned}
$$


as desired.

Having the above lemmas, we are ready to give the proofs of Theorems 1.4 and 1.5.

Proof of Theorem 1.4. Let $\Phi_{1}, \Phi_{2}, \widetilde{\Phi}_{1}, \widetilde{\Phi}_{2} \in \mathcal{A}(\mathbb{R})$ be even functions satisfying

$$
\Phi_{1}(0)=\Phi_{2}(0)=\widetilde{\Phi}_{1}(0)=\widetilde{\Phi}_{2}(0)=0 .
$$

Let $p \in(0, \infty)$ and $\lambda_{i}>\left(n_{i}+D_{i}\right) q_{w} / \min \{p, 2\}, i=1,2$. Note that for a.e. $\left(x_{1}, x_{2}\right) \in$ $X_{1} \times X_{2}$,

$$
\widetilde{\Phi}_{1}\left(t_{1} \sqrt{L_{1}}\right) \otimes \widetilde{\Phi}_{2}\left(t_{2} \sqrt{L_{2}}\right) f\left(x_{1}, x_{2}\right) \leq\left[\widetilde{\Phi}_{1}\left(t_{1} \sqrt{L_{1}}\right) \otimes \widetilde{\Phi}_{2}\left(t_{2} \sqrt{L_{2}}\right)\right]_{\lambda_{1}, \lambda_{2}}^{*} f\left(x_{1}, x_{2}\right) .
$$

Using (3.17), Lemma 3.3 and Lemma 3.5, we infer

$$
\begin{aligned}
& \left\|g_{\widetilde{\Phi}_{1}, \widetilde{\Phi}_{2}, L_{1}, L_{2}}(f)\right\|_{L_{w}^{p}\left(X_{1} \times X_{2}\right)} \\
& \quad=\left\|\left(\int_{0}^{\infty} \int_{0}^{\infty}\left|\widetilde{\Phi}_{1}\left(t_{1} \sqrt{L_{1}}\right) \otimes \widetilde{\Phi}_{2}\left(t_{2} \sqrt{L_{2}}\right) f\right|^{2} \frac{d t_{1}}{t_{1}} \frac{d t_{2}}{t_{2}}\right)^{1 / 2}\right\|_{L_{w}^{p}\left(X_{1} \times X_{2}\right)} \\
& \quad \leq\left\|\left(\int_{0}^{\infty} \int_{0}^{\infty}\left|\left[\widetilde{\Phi}_{1}\left(t_{1} \sqrt{L_{1}}\right) \otimes \widetilde{\Phi}_{2}\left(t_{2} \sqrt{L_{2}}\right)\right]_{\lambda_{1}, \lambda_{2}}^{*} f\right|^{2} \frac{d t_{1}}{t_{1}} \frac{d t_{2}}{t_{2}}\right)^{1 / 2}\right\|_{L_{w}^{p}\left(X_{1} \times X_{2}\right)} \\
& \quad \leq C\left\|\left(\int_{0}^{\infty} \int_{0}^{\infty}\left|\left[\Phi_{1}\left(t_{1} \sqrt{L_{1}}\right) \otimes \Phi_{2}\left(t_{2} \sqrt{L_{2}}\right)\right]_{\lambda_{1}, \lambda_{2}}^{*} f\right|^{2} \frac{d t_{1}}{t_{1}} \frac{d t_{2}}{t_{2}}\right)^{1 / 2}\right\|_{L_{w}^{p}\left(X_{1} \times X_{2}\right)} \\
& \quad \leq C\left\|\left(\int_{0}^{\infty} \int_{0}^{\infty}\left|\Phi_{1}\left(t_{1} \sqrt{L_{1}}\right) \otimes \Phi_{2}\left(t_{2} \sqrt{L_{2}}\right) f\right|^{2} \frac{d t_{1}}{t_{1}} \frac{d t_{2}}{t_{2}}\right)^{1 / 2}\right\|_{L_{w}^{p}\left(X_{1} \times X_{2}\right)} \\
& \quad=C\left\|g_{\Phi_{1}, \Phi_{2}, L_{1}, L_{2}}(f)\right\|_{L_{w}^{p}\left(X_{1} \times X_{2}\right)} .
\end{aligned}
$$

By symmetry, there also holds that

$$
\left\|g_{\Phi_{1}, \Phi_{2}, L_{1}, L_{2}}(f)\right\|_{L_{w}^{p}\left(X_{1} \times X_{2}\right)} \leq C\left\|g_{\Psi_{1}, \Psi_{2}, L_{1}, L_{2}}(f)\right\|_{L_{w}^{p}\left(X_{1} \times X_{2}\right)} .
$$

Hence the assertion of Theorem 1.4 is true.

Proof of Theorem 1.5. Let $\Phi_{1}, \Phi_{2} \in \mathcal{A}(\mathbb{R})$ be even functions. Let $p \in(0, \infty)$, $\lambda_{i}>2 q_{w} / \min \{p, 2\}$ and $\lambda_{i}^{\prime}>\left(n_{i}+D_{i}\right) q_{w} / \min \{p, 2\}, i=1,2$. Then, for all $f \in L^{2}\left(X_{1} \times\right.$ $X_{2}$ ), by (3.17), Lemma 3.6, Lemma 3.1, Lemma 3.2 and Lemma 3.5, we have

$$
\begin{aligned}
& \left\|g_{\Phi_{1}, \Phi_{2}, L_{1}, L_{2}}(f)\right\|_{L_{w}^{p}\left(X_{1} \times X_{2}\right)} \\
& \leq \|\left(\int_{0}^{\infty} \int_{0}^{\infty}\left|\left[\Phi_{1}\left(t_{1} \sqrt{L_{1}}\right) \otimes \Phi_{2}\left(t_{2} \sqrt{L_{2}}\right)\right]_{\left(n_{1} / 2\right) \lambda_{1}+D_{1} / 2,\left(n_{2} / 2\right) \lambda_{2}+D_{2} / 2}^{*} f\right|^{2}\right. \\
& \left.\times \frac{d t_{1}}{t_{1}} \frac{d t_{2}}{t_{2}}\right)^{1 / 2} \|_{L_{w}^{p}\left(X_{1} \times X_{2}\right)} \\
& \leq C\left\|g_{\Phi_{1}, \Phi_{2}, L_{1}, L_{2}, \lambda_{1}, \lambda_{2}}^{*}(f)\right\|_{L_{w}^{p}\left(X_{1} \times X_{2}\right)}
\end{aligned}
$$




$$
\begin{aligned}
& \leq C\left\|S_{\Phi_{1}, \Phi_{2}, L_{1}, L_{2}}(f)\right\|_{L_{w}^{p}\left(X_{1} \times X_{2}\right)} \\
& \leq C\left\|\left(\int_{0}^{\infty} \int_{0}^{\infty}\left|\left[\Phi_{1}\left(t_{1} \sqrt{L_{1}}\right) \otimes \Phi_{2}\left(t_{2} \sqrt{L_{2}}\right)\right]_{\lambda_{1}^{\prime}, \lambda_{2}^{\prime}}^{*} f\right|^{2} \frac{d t_{1}}{t_{1}} \frac{d t_{2}}{t_{2}}\right)^{1 / 2}\right\|_{L_{w}^{p}\left(X_{1} \times X_{2}\right)} \\
& \leq C\left\|g_{\Phi_{1}, \Phi_{2}, L_{1}, L_{2}}(f)\right\|_{L_{w}^{p}\left(X_{1} \times X_{2}\right)},
\end{aligned}
$$

which yields (1.5). The proof of Theorem 1.5 is complete.

\section{Applications of Theorems 1.4 and $\mathbf{1 . 5}$.}

1. In [7] and [11], the theory of product Hardy space $H_{L_{1}, L_{2}}^{1}\left(\mathbb{R}^{n} \times \mathbb{R}^{m}\right)$ via the Littlewood-Paley area functions was established, where $L_{1}$ and $L_{2}$ are two nonnegative self-adjoint operators that satisfy only the Gaussian heat kernel bound. To be more specific, $H_{L_{1}, L_{2}}^{1}\left(\mathbb{R}^{n} \times \mathbb{R}^{m}\right)$ is defined as the closure of

$$
\left\{f \in L^{2}\left(\mathbb{R}^{n} \times \mathbb{R}^{m}\right): S_{L_{1}, L_{2}}(f) \in L^{1}\left(\mathbb{R}^{n} \times \mathbb{R}^{m}\right)\right\}
$$

under the norm $\|f\|_{H_{L_{1}, L_{2}}^{1}\left(\mathbb{R}^{n} \times \mathbb{R}^{m}\right)}:=\left\|S_{L_{1}, L_{2}}(f)\right\|_{H_{L_{1}, L_{2}}^{1}\left(\mathbb{R}^{n} \times \mathbb{R}^{m}\right)}$, where

$$
\begin{aligned}
& S_{L_{1}, L_{2}}(f)\left(x_{1}, x_{2}\right) \\
& \quad:=\left(\iint_{\Gamma_{1}\left(x_{1}\right) \times \Gamma_{2}\left(x_{2}\right)}\left|\left(t_{1}^{2} L_{1} e^{-t_{1}^{2} L_{1}}\right) \otimes\left(t_{2}^{2} L_{2} e^{-t_{2}^{2} L_{2}}\right) f\left(y_{1}, y_{2}\right)\right|^{2} \frac{d y_{1} d t_{1}}{t_{1}^{n+1}} \frac{d y_{2} d t_{2}}{t_{2}^{m+1}}\right)^{1 / 2} .
\end{aligned}
$$

Then, by applying our main result Theorem 1.5 (also Remark 1.7), we obtain the characterization of $H_{L_{1}, L_{2}}^{1}\left(\mathbb{R}^{n} \times \mathbb{R}^{m}\right)$ via the Littlewood-Paley square function as follows, which is missing in [7] and [11], i.e., $H_{L_{1}, L_{2}}^{1}\left(\mathbb{R}^{n} \times \mathbb{R}^{m}\right)$ is equivalent to the closure of

$$
\left\{f \in L^{2}\left(\mathbb{R}^{n} \times \mathbb{R}^{m}\right): g_{L_{1}, L_{2}}(f) \in L^{1}\left(\mathbb{R}^{n} \times \mathbb{R}^{m}\right)\right\}
$$

under the norm $\left\|g_{L_{1}, L_{2}}(f)\right\|_{H_{L_{1}, L_{2}}^{1}\left(\mathbb{R}^{n} \times \mathbb{R}^{m}\right)}$, where

$$
g_{L_{1}, L_{2}}(f)\left(x_{1}, x_{2}\right)=\left(\int_{0}^{\infty} \int_{0}^{\infty}\left|\left(t_{1}^{2} L_{1} e^{-t_{1}^{2} L_{1}}\right) \otimes\left(t_{2}^{2} L_{2} e^{-t_{2}^{2} L_{2}}\right) f\left(x_{1}, x_{2}\right)\right|^{2} \frac{d t_{1}}{t_{1}} \frac{d t_{2}}{t_{2}}\right)^{1 / 2} .
$$

2. In 1965, Muckenhoupt and Stein in [17] introduced a notion of conjugacy associated with the Bessel operator $\Delta_{\lambda}$ on $\mathbb{R}_{+}:=(0, \infty)$ defined by

$$
\Delta_{\lambda} f(x):=-\frac{d^{2}}{d x^{2}} f(x)-\frac{2 \lambda}{x} \frac{d}{d x} f(x), \quad x>0,
$$

and the Bessel Schrödinger operator $S_{\lambda}$ on $\mathbb{R}_{+}$

$$
S_{\lambda} f(x):=-\frac{d^{2}}{d x^{2}} f(x)+\frac{\lambda^{2}-\lambda}{x^{2}} f(x), \quad x>0 .
$$

In [9], Duong et al. established the product Hardy space $H_{\Delta_{\lambda}}^{p}\left(\mathbb{R}_{+} \times \mathbb{R}_{+}\right)$associated with $\Delta_{\lambda}$ via the Littlewood-Paley area function and square functions. Note that the 
measure on $\mathbb{R}_{+}$related to $\Delta_{\lambda}$ is $d \mu_{\lambda}(x)=x^{2 \lambda} d x$. We point out that the kernel of $t^{2} \Delta_{\lambda} e^{-t^{2} \Delta_{\lambda}}$ satisfies the Gaussian upper bounds with respect to the measure $d \mu_{\lambda}$, the Hölder regularity and the cancellation property. Hence, by using the approach in [15] via the Plancherel-Polya type inequality, they obtained the equivalence of the characterizations of $H_{\Delta_{\lambda}}^{p}\left(\mathbb{R}_{+} \times \mathbb{R}_{+}\right)$via Littlewood-Paley area function and square functions. By applying our main result Theorem 1.5 (also Remark 1.7), we obtain a direct proof of the equivalence without using the Hölder regularity and the cancellation property.

In $[\mathbf{1}]$, Betancor et al. established the product Hardy space $H_{S_{\lambda}}^{p}\left(\mathbb{R}_{+} \times \mathbb{R}_{+}\right)$associated with $\Delta_{\lambda}$ via the Littlewood-Paley area function and square functions. To prove the equivalence, they need to use the Poisson semigroup $\left\{e^{-t \sqrt{S_{\lambda}}}\right\}$, the subordination formula and the Moser type inequality as a bridge. By applying our main result Theorem 1.5 (also Remark 1.7), we obtain a direct proof of this equivalence without using the Moser type inequality.

AcKnowledgements. The authors would like to thank the referee for the careful reading and comments, which made this paper more accurate. The authors also thank the editors for their patience and helpful suggestions.

\section{References}

[1] J. J. Betancor, X. T. Duong, J. Li, B. D. Wick and D. Yang, Product Hardy, BMO spaces and iterated commutators associated with Bessel Schrödinger operators, to appear in Indiana Univ. Math. J.

[2] H.-Q. Bui, M. Paluszyński and M. H. Taibleson, A maximal function characterization of BesovLipschitz and Triebel-Lizorkin spaces, Studia Math., 119 (1996), 219-246.

[3] H.-Q. Bui, M. Paluszyński and M. H. Taibleson, Characterization of the Besov-Lipschitz and Triebel-Lizorkin spaces: The case $q<1$, J. Fourier Anal. Appl., 3 (1997), 837-846.

[4] F. Cacciafesta and P. D'Ancona, Weighted $L^{p}$ estimates for powers of selfadjoint operators, Adv. Math., 229 (2012), 501-530.

[5] S.-Y. A. Chang and R. Fefferman, A continuous version of the duality of $H^{1}$ with BMO on the bi-disc, Ann. of Math. (2), 112 (1980), 179-201.

[6] D. Deng and Y. Han, Harmonic analysis on spaces of homogeneous type, with a preface by Yves Meyer, Lecture Notes in Math., 1966, Springer-Verlag, Berlin, 2009.

[ 7 ] D. Deng, L. Song, C. Tan and L. Yan, Duality of Hardy and BMO spaces associated with operators with heat kernel bounds on product domains, J. Geom. Anal., 17 (2007), 455-483.

[8] X. T. Duong, J. Li, L. Song and L. Yan, Frame decomposition and radial maximal semigroup characterization of Hardy spaces associated to operators, submitted 2016.

[ 9 ] X. T. Duong, J. Li, B. D. Wick and D. Yang, Characterizations of product Hardy spaces in Bessel setting, arXiv:1606.03500.

[10] X. T. Duong, J. Li and L. Yan, A Littlewood-Paley type decomposition and weighted Hardy spaces associated with operators, J. Geom. Anal., 26 (2016), 1617-1646.

[11] X. T. Duong, J. Li and L. Yan, End-point estimates for singular integrals with non-smooth kernels on product spaces, arXiv:1509.07548.

[12] C. Fefferman and E. M. Stein, $H^{p}$ spaces of several variables, Acta Math., 129 (1972), 137-193.

[13] R. Fefferman, Harmonic analysis on product spaces, Ann. of Math. (2), 126 (1987), 109-130.

[14] J. García-Cuerva and J. L. Rubio de Francia, Weighted Norm Inequalities and Related Topics, North-Holland, Amsterdam, 1985.

[15] Y. Han, J. Li and L. A. Ward, Hardy space theory on spaces of homogeneous type via orthonormal wavelet bases, Appl. Comput. Harmon. Anal., 45 (2018), 120-169.

[16] G. Hu, Littlewood-Paley characterization of weighted Hardy spaces associated with operators, J. Aust. Math. Soc., 103 (2017), 250-267. 
[17] B. Muckenhoupt and E. M. Stein, Classical expansions and their relation to conjugate harmonic functions, Trans. Amer. Math. Soc., 118 (1965), 17-92.

[18] V. S. Rychkov, On a theorem of Bui, Paluszyński and Taibleson, Proc. Steklov Inst., 227 (1999), 280-292.

[19] S. Sato, Weighted inequalities on product domains, Studia Math., 92 (1989), 59-72.

[20] L. Song and L. Yan, Maximal function characterizations for Hardy spaces associated to nonnegative self-adjoint operators on spaces of homogeneous type, to appear in J. Evolution Equations.

[21] E. M. Stein, Harmonic Analysis: Real-Variable Methods, Orthogonality, and Oscillatory Integrals, Princeton University Press, Princeton, NJ, 1993.

[22] J.-O. Strömberg and A. Torchinsky, Weighted Hardy spaces, Lecture Notes in Math., 1381, Springer-Verlag, Berlin, 1989.

\section{Xuan Thinh Duong}

Department of Mathematics

Macquarie University

Sydney

NSW 2109, Australia

E-mail: xuan.duong@mq.edu.au

\section{Guorong Hu}

Department of Mathematics Jiangxi Normal University Nanchang Jiangxi 330022, China

E-mail: hugr@mail.ustc.edu.cn

\section{Ji LI}

Department of Mathematics Macquarie University

Sydney

NSW 2109, Australia

E-mail: ji.li@mq.edu.au 